\title{
One parameter family of rationally extended isospectral potentials
}

\author{
Rajesh Kumar Yadav ${ }^{a *}$, Suman Banerjee ${ }^{a \dagger}$, Nisha Kumari $^{b \ddagger}$ Avinash Khare ${ }^{c \S}$, and \\ Bhabani Prasad Mandal ${ }^{d}$
}

April 29, 2020

\author{
${ }^{a}$ Department of Physics, S. K. M. University, Dumka-814110, INDIA. \\ ${ }^{b}$ Department of Physics, S. P. College, Dumka-814101, INDIA. \\ ${ }^{c}$ Department of Physics, Savitribai Phule Pune University, Pune-411007, INDIA. \\ ${ }^{d}$ Department of Physics, Banaras Hindu University, Varanasi-221005, INDIA.
}

\begin{abstract}
We start from a given one dimensional rationally extended potential associated with $X_{m}$ exceptional orthogonal polynomials and using the idea of supersymmetry in quantum mechanics, we obtain one continuous parameter $(\lambda)$ family of rationally extended strictly isospectral potentials whose solutions are also associated with $X_{m}$ exceptional orthogonal polynomials. We illustrate this construction by considering three well known rationally extended potentials, two with pure discrete spectrum (the extended radial oscillator and the extended Scarf-I) and one with both the discrete and the continuous spectrum (the extended generalized Pöschl-Teller) and explicitly construct the corresponding one continuous parameter family of rationally extended strictly isospectral potentials. Further, in the special case of $\lambda=0$ and -1 , we obtain two new exactly solvable rationally extended potentials, namely the rationally extended Pursey and the rationally extended Abhrahm-Moses potentials respectively. We illustrate the whole procedure by discussing in detail the particular case of the $X_{1}$ rationally extended one parameter family of potentials including the corresponding Pursey and the Abraham Moses potentials.
\end{abstract}

\footnotetext{
*e-mail address: rajeshastrophysics@gmail.com (R.K.Y)

$\dagger$ e-mail address: suman.raghunathpur@gmail.com (S.B)

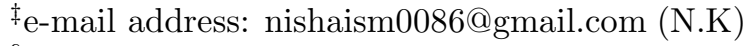

$\S$ e-mail address: khare@physics.univpune.ac.in (A.K)

ฯe-mail address: bhabani.mandal@gmail.com (B.P.M).
} 


\section{Introduction}

Since the advent of supersymmetric (SUSY) quantum mechanics [1, 2] there has been a flood of activity in discovering new exactly solvable potentials. Amongst these, special mention may be made of isospectral potentials which have application in several diverse areas like in the problems of inverse scattering [3, 4], $\alpha \alpha$ - scattering [5], soliton theory [6, 7] etc. This has motivated the researchers to search for a family of isospectral potentials [8, 9, 10, 11, 12. Several different approaches have been used for this purpose such as Darboux method [13, Abraham and Moses method [12, Pursey method [10, and Supersymmetric (SUSY) method [14] etc to discover new isospectral potentials. In a very interesting paper Pursey [10] has shown that the first three methods are inequivalent in the case of inserting or deleting states while for normalizing states these are equivalent to that of Abraham and Moses approach (which is based on Gelfand-Levitan approach [15]). Subsequently, Amado [9] followed the Pursey approach and constructed the potentials isospectral to the coulomb potential with one less bound state. Out of all these approaches, the SUSY approach is simpler and convenient in searching for a family of isospectral potentials. In particular, using this approach it was shown [8, 14] that for any given central or one dimensional potential with at least one bound state, a continuous oneparameter $(\lambda)$ family of strictly isospectral potentials can be obtained. They further showed that as $\lambda \rightarrow 0$, the potential reduces to the Pursey potential [10] and as $\lambda \rightarrow-1$, it reduces to the Abraham-Moses [AM] potential [12]. It has also been shown that the partner potential has the same bound state spectrum as those of the Pursey and the AM potentials. They considered the usual Coulomb and the radial oscillator potentials and discussed the various isospectral potentials following from them.

Recently, after the discovery of the $X_{m}$-exceptional orthogonal polynomials (EOPs) $(m \geq 1)$ [16, 17, 18], many of the well known shape invariant potentials have been extended rationally and their exact solutions have been obtained in terms of these EOPs [19, 20, 21, 22, 23, 24, 25, 26, 27, 28, 29]. Using supersymmetric (SUSY) quantum mechanics [1, 2], it has been shown that these rationally extended (RE) potentials are also shape invariant. Various properties of these extended potentials have been studied in [30, 31, 32, 33, 34, 35, 36, 37, 38, 39, 40, 41, 42, 43, 44]. It is worth pointing out that the corresponding (non-rational) potentials are the special $m=0$ case of the related RE potentials.

One interesting question is how to extend the number of analytically solvable RE potentials. In this context, we observe that to the best of our knowledge so far one continuous parameter family of strictly isospectral potentials (i.e. those with identical discrete spectrum and identical reflection and transmission amplitudes in the one dimensional case or identical scattering amplitude in the three dimensional case) have only been obtained for the case of the conventional (non-rational) potentials, but no such construction exists for the RE potentials. Similarly, while Pursey and AM potentials corresponding to the usual (non-rational) potentials are known, the corresponding rational Pursey and AM potentials are still not available. The purpose of this paper is to fill this gap and in this process also extend the number of analytically solvable isospectral RE potentials. 
In this paper, we consider RE potentials and follow the formalism of SUSY quantum mechanics and generate one continuous parameter $(\lambda)$ family of exactly solvable RE strictly isospectral potentials. In the limit of $\lambda=0$ and $\lambda=-1$, we then obtain the corresponding RE Pursey and the RE AM potentials respectively. We elaborate this construction by considering three well known RE potentials, two with pure discrete spectrum (i.e. the extended radial oscillator and the Scarf-I potentials) and one with discrete as well as the continuous spectrum (i.e. the extended generalized Pöschl-Teller potential) and for all three cases construct the corresponding one parameter family of strictly isospectral $\mathrm{RE}$ potentials. In other words, if we start from these three conventional (non-rational) potentials then one has constructed two parameter family of strictly isospectral potentials. Out of these two, $\lambda$ is a continuous parameter while $m$ is a discrete parameter $(m=1,2,3, \ldots)$ corresponding to $X_{m}$ EOPs. Further, we also obtain the corresponding one discrete parameter $m$ family of RE Pursey and RE AM potentials.

The organization of this paper is as follows: In Sec. 2 we briefly discuss the SUSY formalism and explain how by starting from any potential with at least one bound state one can construct one continuous parameter $(\lambda)$ family of strictly isospectral potentials. Further we also point out how to obtain the corresponding Pursey and the AM potentials and explain how their eigenfunctios are related to those of the original potential with at least one bound state. In Sec. 3, we consider three RE potentials associated with the $X_{m}$ EOPs and construct the corresponding one continuous parameter $(\lambda)$ family of the RE strictly isospectral potentials $\hat{V}_{m}^{(-)}(x, \lambda)$ with their solutions in terms of $X_{m}$ EOPs. For illustration, we consider the $X_{1}(m=1)$ case in detail for all three RE potentials and give plots of $\hat{V}_{1}^{(-)}(x, \lambda)$ for several values of $\lambda$ as well as the corresponding ground state eigenfunctions for all three RE potentials. Further, we also give plots of the corresponding three isospectral potentials $V_{1}^{[+]}, V_{1}^{[P]}$ and $V_{1}^{[A M]}$. Finally, we summarize our results in Sec. 4 .

\section{Supersymmetric (SUSY) Quantum Mechanics for- malism}

In this section, we briefly review the SUSY QM formalism [14] to construct one continuous parameter $(\lambda)$ family of strictly isospectral potentials corresponding to any potential with at least one bound state. Given a Hamiltonian with ground state energy $E_{0}$, one can trivially construct a new Hamiltonian from here with zero ground state energy. In the SQM formalism one considers a Hamiltonian $(\hbar=2 m=1)$

$$
H^{(-)}(x)=-\frac{d^{2}}{d x^{2}}+V^{(-)}(x),
$$

with zero ground state energy and the corresponding ground state eigenfunction being $\psi_{0}^{(-)}(x)$. It can be factorized in terms of operators $A$ and $A^{\dagger}$ as

$$
H^{(-)}(x)=A^{\dagger} A
$$


with

$$
A=\frac{d}{d x}+W(x) \quad \text { and } \quad A^{\dagger}=-\frac{d}{d x}+W(x),
$$

where

$$
W(x)=-\frac{d}{d x}\left[\ln \psi_{0}^{(-)}(x)\right]
$$

is the superpotential, which determines the two partner potentials

$$
V^{( \pm)}(x)=W^{2}(x) \pm W^{\prime}(x) .
$$

The eigenvalues and the eigenfunctions of these two partner potentials (when the SUSY is unbroken) are related by

$$
E_{n+1}^{(-)}=E_{n}^{(+)} \quad E_{0}^{(-)}=0
$$

and

$$
\psi_{n}^{(+)}(x)=\frac{1}{\left[E_{n}^{(+)}\right]^{1 / 2}} A \psi_{n+1}^{(-)} \quad \psi_{n+1}^{(-)}(x)=\frac{1}{\left[E_{n}^{(+)}\right]^{1 / 2}} A^{\dagger} \psi_{n}^{(+)}
$$

respectively. For the one dimensional case, the transmission $\left(t^{( \pm)}(k)\right)$ and reflection $\left(r^{( \pm)}(k)\right)$ amplitudes for the partner potentials $V^{( \pm)}(x)$ are related by

$$
r^{(-)}(k)=\left(\frac{W_{-}+i k}{W_{-}-i k}\right) r^{(+)}(k)
$$

and

$$
t^{(-)}(k)=\left(\frac{W_{+}-i k^{\prime}}{W_{-}-i k}\right) t^{(+)}(k)
$$

where

$$
k=\left(E-W_{-}^{2}\right)^{\frac{1}{2}} \quad \text { and } \quad k^{\prime}=\left(E-W_{+}^{2}\right)^{\frac{1}{2}}
$$

with

$$
W_{ \pm}=W(x \rightarrow \pm \infty) .
$$

Similarly, for $3 D$-central potentials the scattering amplitudes for the partner potentials are related by [1]

$$
s^{(-)}\left(k^{\prime}\right)=\left(\frac{W_{+}-i k^{\prime}}{W_{+}+i k^{\prime}}\right) s^{(+)}\left(k^{\prime}\right) .
$$

\subsection{Generation of isospectral potentials}

The one-parameter family of potentials $\hat{V}^{(-)}(\lambda, x)$ which are strictly isospectral to a given potential $V^{(-)}(x)$ are obtained by considering the uniqueness of the partner potential $V^{(+)}(x)$ [14], i.e. for a given more general form of the superpotential $\hat{W}(x), V^{+}(x)$ satisfies

$$
V^{(+)}(x)=\hat{W}^{2}(x)+\hat{W}^{\prime}(x)=W^{2}(x)+W^{\prime}(x) .
$$


Here $\hat{W}(x)=W(x)$ is one of the solution of the above equation. The most general solution to the Eq. (13) can be obtained by defining

$$
\hat{W}(x)=W(x)+\phi(x) .
$$

On using (14) in (13) and compare with (15), we get

$$
\phi^{\prime}(x)+2 W(x) \phi(x)+\phi^{\prime}(x)=0 .
$$

If we set $y(x)=\frac{1}{\phi(x)}$, we get the Bernoulli equation

$$
y^{\prime}(x)=1+2 W(x) y(x) .
$$

with the solution

$$
\frac{1}{y(x)}=\phi(x)=\frac{d}{d x} \ln [I(x)+\lambda]
$$

where the integral $I(x)$, in term of the normalized ground state wavefunction, is given by

$$
I(x)=\int_{-\infty}^{x}\left[\psi_{0}^{(-)}(x)\right]^{2} d x
$$

and $\lambda$ is an abitrary real constant. Thus the most general form of the superpotential $\hat{W}(x)$ satisfying Eq. (13) is given by

$$
\hat{W}(x)=W(x)+\frac{d}{d x} \ln [I(x)+\lambda] .
$$

Thus one obtains one continuous parameter family of potentials

$$
\hat{V}^{(-)}(\lambda, x)=\hat{W}^{2}(x)-\hat{W}^{\prime}(x)=V^{(-)}(x)-2 \frac{d^{2}}{d x^{2}} \ln (I(x)+\lambda),
$$

all of which have the same SUSY partner potential $V^{(+)}(x)$. From Eqs. (4) and (19), the associated normalized ground state eigenfunction for the potential $\hat{V}^{(-)}(\lambda, x)$ is given by

$$
\hat{\psi}_{0}^{(-)}(\lambda, x)=\frac{\sqrt{\lambda(1+\lambda)} \psi_{0}^{(-)}(x)}{[I(x)+\lambda]}
$$

and from Eqs. (77) and (19) the normalized excited-state $(n=0,1,2, \ldots)$ eigenfunctions are

$$
\hat{\psi}_{n+1}^{(-)}(\lambda, x)=\psi_{n+1}^{(-)}(x)+\frac{1}{E_{n+1}^{(-)}}\left(\frac{I^{\prime}(x)}{I(x)+\lambda}\right)\left(\frac{d}{d x}+W(x)\right) \psi_{n+1}^{(-)}(x) .
$$

The associated energy eigenvalues are

$$
\hat{E}_{n+1}^{(-)}=E_{n}^{(+)}, \quad E_{0}^{(-)}=0, \quad n=1,2, \ldots .
$$


From Eqs. (18) and (21) it is clear that the ground state eigenfunction is acceptable only if $\lambda>0$ or $\lambda<-1$ and in these cases the entire one continuous parameter family of potentials have the same energy eigenvalues as $V^{(-)}(x)$. Besides, in case $\lambda>0$ or $\lambda<-1$

$$
\hat{W}(x \rightarrow \pm \infty)=W(x \rightarrow \pm \infty)=W_{ \pm}
$$

and hence in these cases the scattering amplitudes are also the same as those of $V^{(-)}(x)$ i.e. are given by Eqs. (8) and (9) in the one dimensional case or by Eq. (11) in the three dimensional case. Summarizing, given any potential $V^{(-)}(x)$ with at least one bound state, one can easily construct one continuous parameter family of potentials $V^{(-)}(x, \lambda)$ which are strictly isospectral to the potential $V^{(-)}(x)$ provided $\lambda>0$ or $\lambda<-1$.

On the other hand when $\lambda=0$ or $\lambda=-1$, then $I(x)+\lambda$ with $I(x)$ given by Eq. (18) will vanish either at $x \rightarrow-\infty$ or $x \rightarrow+\infty$ respectively, so that the ground state eigenfunction $\hat{\psi}_{0}^{(-)}(\lambda, x)$ as given by Eq. (21) is no more square integrable and hence the SUSY is broken so that the bound state spectrum is degenerate with the partner potential $V^{(+)}(x)$, i.e. in this case the potential $\hat{V}^{(-)}(\lambda, x)$ loses a bound state. The potentials corresponding to $\lambda=0$ and -1 are the so called Pursey $V^{[P]}(x)$ and the Abraham-Moses $V^{[A M]}(x)$ potentials respectively [14]. For completeness we now mention some of the important results of these two potentials.

\subsubsection{The Pursey potential}

The superpotential for this case is defined by putting $\lambda=0$ in Eq. (201)

$$
W^{[P]}(x)=W(x)+\frac{d}{d x} \ln I(x) .
$$

and the potential (201) becomes

$$
V^{[P]}(x)=\hat{V}^{(-)}(\lambda=0, x)=V^{(-)}(x)-2 \frac{d^{2}}{d x^{2}} \ln I(x) .
$$

Since the supersymmetry between $V^{(+)}(x)$ and $V^{[P]}(x)$ is broken, thus the energy eigenvalues of this potential are isospectral to those of $V^{(+)}(x)$, i.e.

$$
E_{n}^{[P]}=E_{n}^{(+)} .
$$

Further, the scattering amplitudes for one dimensional case are

$$
\begin{aligned}
& r^{[p]}(k)=\left(\frac{W_{-}-i k}{W_{-}+i k}\right)^{2} r^{(-)}(k) \\
& t^{[p]}(k)=-\left(\frac{W_{-}-i k}{W_{-}+i k}\right) t^{(-)}(k)
\end{aligned}
$$


while for the three dimensional case

$$
s^{[P]}\left(k^{\prime}\right)=s^{(-)}\left(k^{\prime}\right) .
$$

The normalized eigenfunctions $\psi_{n}^{[P]}(x)$ for the Pursey potentual $V^{[P]}(x)$ are obtained by putting $\lambda=0$ in Eq. (22) i.e.,

$$
\begin{aligned}
\psi_{n}^{[p]}(x) & =\hat{\psi}_{n+1}^{(-)}(\lambda=0, x) \\
& =\psi_{n+1}^{(-)}(x)+\frac{1}{E_{n+1}^{(-)}}\left(\frac{I^{\prime}(x)}{I(x)}\right)\left(\frac{d}{d x}+W(x)\right) \psi_{n+1}^{(-)}(x),
\end{aligned}
$$

where $n=0,1,2 \ldots$, .

\subsubsection{The Abraham-Moses potential}

In this case since $\lambda=-1$, the superpotential and the potential are given by

$$
W^{[A M]}(x)=W(x)+\frac{d}{d x} \ln (I(x)-1),
$$

and

$$
V^{[A M]}(x)=\hat{V}^{(-)}(\lambda=-1, x)=V^{(-)}(x)-2 \frac{d^{2}}{d x^{2}} \ln (I(x)-1) .
$$

Thus as in the Pursey case, the supersymmetry between $V^{(+)}(x)$ and $V^{[P]}(x)$ is broken, thus the energy eigenvalues will be

$$
E_{n}^{[A M]}=E_{n}^{(+)}
$$

Further, the scattering amplitudes in one dimensional cases are

$$
\begin{gathered}
r^{[A M]}(k)=r^{(-)}(k) \\
t^{[A M]}(k)=-\left(\frac{W_{+}+i k^{\prime}}{W_{+}-i k^{\prime}}\right) t^{(-)}(k)
\end{gathered}
$$

while in the three dimensional case

$$
s^{[A M]}\left(k^{\prime}\right)=\left(\frac{W_{+}+i k^{\prime}}{W_{+}-i k^{\prime}}\right)^{2} s^{(-)}\left(k^{\prime}\right) .
$$

In this case, the normalized eigenfunctions $\psi_{n}^{[A M]}(x)(n=0,1,2, \ldots)$ for the AM potentual $V^{[A M]}(x)$ are obtained by putting $\lambda=-1$ in Eq. (22) , i.e.

$$
\begin{aligned}
\psi_{n}^{[A M]}(x) & =\hat{\psi}_{n+1}^{(-)}(\lambda=-1, x) \\
& =\psi_{n+1}^{(-)}(x)+\frac{1}{E_{n+1}^{(-)}}\left(\frac{I^{\prime}(x)}{I(x)-1}\right)\left(\frac{d}{d x}+W(x)\right) \psi_{n+1}^{(-)}(x) .
\end{aligned}
$$


Summarizing, given any potential $V^{(-)}(x)$ with at least one bound state, one can always construct three potentials $V^{(+)}, V^{[P]}(x), V^{[A M]}(x)$ which are isospctral to each other but may or may not be strictly isospectral, i.e. while their energy eigenvalues are the same, their reflection and transmission amplitudes in the one dimensional case or scattering amplitude in the three dimensional case may or may not be the same. For example, for potentials with purely discrete spectrum, obviously they are strictly isospectral.

\section{Rationally extended isospectral potentials}

In this section, we consider three examples of RE exactly solvable shape invariant potentials (namely the extended radial oscillator, extended Scarf-I and extended generalized Pöschl-Teller potentials) whose solutions are associated with the $X_{m}$ Laguerre (extended radial oscillator) and $X_{m}$ Jacobi (extended Scarf-I and generalized Pöschl-Teller potentials)EOPs [21, 28, 29] and in all three cases obtain explicitly the corresponding rationally extended one-parameter family of isospectral potentials. Since we are considering the RE potentials which are $m$-dependent (the corresponding eigenfunctions being $X_{m}$ Laguerre or $X_{m}$ Jacobi polynomials), hence we introduce a subscript $m$ with all the terms defined in the last section such as $W(x) \Rightarrow W_{m}(x), V^{( \pm)}(x) \Rightarrow V_{m}^{( \pm)}(x), I(x) \Rightarrow I_{m}(x)$ etc. For all these extended potentials, as is well known, the total superpotential is the sum of the superpotential of the conventional (non-rational) term $\left(W_{c o n}(x)\right)$ and the superpotential due to the rational term $\left(W_{m, r a t}(x)\right)$, i.e.

$$
W_{m}(x)=W_{\text {con }}(x)+W_{m, r a t}(x),
$$

Thus the rationally extended potential becomes

$$
V_{m}^{(-)}(x)=W_{m}^{2}(x)-W_{m}^{\prime}(x)=V_{c o n}^{(-)}(x)+V_{m, \text { rat }}^{(-)}(x),
$$

Here $V_{c o n}^{(-)}(x)$ is the corresponding conventional potential and $V_{m, \text { rat }}^{(-)}(x)$ is the extra piece due to the rational term. Similarly the partner potential $V_{m}^{(+)}(x)$ with the associated usual and rational partner terms is given by

$$
V_{m}^{(+)}(x)=W_{m}^{2}(x)+W_{m}^{\prime}(x)=V_{\text {con }}^{(+)}(x)+V_{m, \text { rat }}^{(+)}(x) .
$$

For $m=0$, the rational superpotential term $W_{m, r a t}(x)=0$ and hence $V_{m, \text { rat }}^{(-)}(x)=0$. Thus the extended potential $V_{m}^{(-)}(x)$ reduces to the conventional potential $V_{c o n}^{(-)}(x)$.

\subsection{Rationally extended radial oscillator potential}

In this case the terms associated with the superpotenial (39) are given by [21, 29]

$$
W_{\text {con }}(r)=\frac{\omega r}{2}-\frac{(\ell+1)}{r}
$$


and

$$
W_{m, r a t}(r)=\omega r\left[\frac{L_{m-1}^{\left(\ell+\frac{1}{2}\right)}\left(-\frac{\omega r^{2}}{2}\right)}{L_{m}^{\left(\ell-\frac{1}{2}\right)}\left(-\frac{\omega r^{2}}{2}\right)}-\frac{L_{m-1}^{\left(\ell+\frac{3}{2}\right)}\left(-\frac{\omega r^{2}}{2}\right)}{L_{m}^{\left(\ell+\frac{1}{2}\right)}\left(-\frac{\omega r^{2}}{2}\right)}\right] .
$$

Thus the terms associated with the RE potential (40) become

$$
V_{\text {con }}^{(-)}(r)=\frac{1}{4} \omega^{2} r^{2}+\frac{\ell(\ell+1)}{r^{2}}-\omega\left(\ell+\frac{3}{2}\right)
$$

and

$$
\begin{aligned}
V_{m, r a t}^{(-)}(r) & =-\omega^{2} r^{2} \frac{L_{m-2}^{\left(\ell+\frac{3}{2}\right)}\left(-\frac{\omega r^{2}}{2}\right)}{L_{m}^{\left(\ell-\frac{1}{2}\right)}\left(-\frac{\omega r^{2}}{2}\right)}+\omega\left(\omega r^{2}+2 \ell-1\right) \frac{L_{m-1}^{\left(\ell+\frac{1}{2}\right)}\left(-\frac{\omega r^{2}}{2}\right)}{L_{m}^{\left(\ell-\frac{1}{2}\right)}\left(-\frac{\omega r^{2}}{2}\right)} \\
& +2 \omega^{2} r^{2}\left(\frac{L_{m-1}^{\left(\ell+\frac{1}{2}\right)}\left(-\frac{\omega r^{2}}{2}\right)}{L_{m}^{\left(\ell-\frac{1}{2}\right)}\left(-\frac{\omega r^{2}}{2}\right)}\right)^{2}-2 m \omega, \quad 0<r<\infty .
\end{aligned}
$$

respectively. Similarly, the terms associated with the partner potential (41) are

$$
V_{\text {con }}^{(+)}(r)=\frac{1}{4} \omega^{2} r^{2}+\frac{(\ell+1)(\ell+2)}{r^{2}}-\omega\left(\ell+\frac{3}{2}\right)
$$

and

$$
\begin{aligned}
V_{m, r a t}^{(+)}(r) & =-\omega^{2} r^{2} \frac{L_{m-2}^{\left(\ell+\frac{5}{2}\right)}\left(-\frac{\omega r^{2}}{2}\right)}{L_{m}^{\left(\ell+\frac{1}{2}\right)}\left(-\frac{\omega r^{2}}{2}\right)}+\omega\left(\omega r^{2}+2 \ell+1\right) \frac{L_{m-1}^{\left(\ell+\frac{3}{2}\right)}\left(-\frac{\omega r^{2}}{2}\right)}{L_{m}^{\left(\ell+\frac{3}{2}\right)}\left(-\frac{\omega r^{2}}{2}\right)} \\
& +2 \omega^{2} r^{2}\left(\frac{L_{m-1}^{\left(\ell+\frac{3}{2}\right)}\left(-\frac{\omega r^{2}}{2}\right)}{L_{m}^{\left(\ell+\frac{1}{2}\right)}\left(-\frac{\omega r^{2}}{2}\right)}\right)^{2}-2 m \omega .
\end{aligned}
$$

The ground state eigenfunction corresponding to the extended potential $V_{m}^{(-)}(r)$ in terms of $X_{m}$ Laguerre polynomials $\hat{L}_{m}^{\left(\ell+\frac{1}{2}\right)}\left(\frac{\omega r^{2}}{2}\right)$ can be obtained using Eq. (44) and one gets

$$
\psi_{0, m}^{(-)}(r)=N_{m}^{\ell} \frac{r^{\ell+1} \exp \left(-\frac{\omega r^{2}}{4}\right)}{L_{m}^{\left(\ell-\frac{1}{2}\right)}\left(-\frac{\omega r^{2}}{2}\right)} \hat{L}_{m}^{\left(\ell+\frac{1}{2}\right)}\left(\frac{\omega r^{2}}{2}\right), \quad m=1,2, \ldots,
$$

where $N_{m}^{\ell}$ is the normalization constant which is given by

$$
N_{m}=\left[\frac{\omega^{\left(\ell+\frac{3}{2}\right)}}{2^{\left(\ell+\frac{1}{2}\right)}\left(\ell+2 m-\frac{1}{2}\right) \Gamma\left(\ell+m-\frac{1}{2}\right)}\right]^{1 / 2} .
$$

The excited state eigenfunctions $(n=0,1,2 .$.$) of V_{m}^{(-)}(r)$ are also explicitly known [21] and given by

$$
\psi_{n, m}^{(-)}(r)=N_{n, m}^{\ell} \frac{r^{\ell+1} \exp \left(-\frac{\omega r^{2}}{4}\right)}{L_{m}^{\left(\ell-\frac{1}{2}\right)}\left(-\frac{\omega r^{2}}{2}\right)} \hat{L}_{n+m}^{\left(\ell+\frac{1}{2}\right)}\left(\frac{\omega r^{2}}{2}\right), \quad m=1,2, \ldots,
$$


and the energy eigenvalues are $(n=0,1,2, .$.

$$
E_{n+1}^{(-)}=E_{n}^{+}=2(n+1) \omega, \quad E_{0}^{-}=0,
$$

with

$$
N_{n, m}^{\ell}=\left[\frac{n ! \omega^{\left(\ell+\frac{3}{2}\right)}}{2^{\left(\ell+\frac{1}{2}\right)}\left(\ell+n+2 m-\frac{1}{2}\right) \Gamma\left(\ell+n+m-\frac{1}{2}\right)}\right]^{1 / 2} .
$$

Hence the eigenfunctions of the partner potentials are

$$
\psi_{n, m}^{(+)}(r)=N_{n, m}^{\ell+1} \frac{r^{\ell+2} \exp \left(-\frac{\omega r^{2}}{4}\right)}{L_{m}^{\left(\ell+\frac{1}{2}\right)}\left(-\frac{\omega r^{2}}{2}\right)} \hat{L}_{n+m}^{\left(\ell+\frac{3}{2}\right)}\left(\frac{\omega r^{2}}{2}\right) .
$$

In terms of the classical Laguerre polynomials the $X_{m}$ Laguerre polynomials can be written as

$$
\hat{L}_{n+m}^{(\alpha)}(z)=L_{m}^{(\alpha)}(-z) L_{n}^{(\alpha-1)}(z)+L_{m}^{(\alpha-1)}(-z) L_{n-1}^{(\alpha)}(z) ; \quad n \geq m .
$$

Using the ground state eigenfunction as give by Eqs. (48) and (49) and the results discussed in the last section, it is straight forward to calculate $I_{m}(r)$ as defined by Eq. (18) and obtain one continuous parameter family of RE strictly isospectral potentials $\hat{V}_{m}^{(-)}(\lambda, r)$ and also the corresponding eigenfunctions $\psi_{n, m}^{(-)}(\lambda, r)$ in terms of $X_{m}$ Laguerre polynomials. In the particular cases of $\lambda=0$ and $\lambda=-1$ one then obtains the extended pursey $V_{m}^{[P]}(r)$ and the extended Abraham-Moses $V_{m}^{[A M]}(r)$ potentials respectively.

We now illustrate this general approach by explicitly discussing the $X_{1}$ case $(m=1)$. In this case the extended potential (40) and the corresponding eigenfunctions (50) are

$$
V_{m=1}^{(-)}(r)=\frac{1}{4} \omega r^{2}+\frac{\ell(\ell+1)}{r^{2}}+4 \omega\left(\frac{1}{\left(\omega r^{2}+2 \ell+1\right)}-\frac{2(2 \ell+1)}{\left(\omega r^{2}+2 \ell+1\right)^{2}}\right)-\omega\left(\ell+\frac{3}{2}\right)
$$

and

$$
\psi_{n, m=1}^{(-)}(r)=N_{n, 1}^{\ell} \frac{r^{\ell+1} \exp \left(-\frac{\omega r^{2}}{4}\right)}{L_{1}^{\left(\ell-\frac{1}{2}\right)}\left(-\frac{\omega r^{2}}{2}\right)} \hat{L}_{n+1}^{\left(\ell+\frac{1}{2}\right)}\left(\frac{\omega r^{2}}{2}\right)
$$

respectively. The energy eigenvalues are the same as that of the conventional case and are given by Eq. (51). Similarly from Eq. (41), the corresponding partner potential is

$$
V_{1}^{(+)}(r)=\frac{1}{4} \omega r^{2}+\frac{(\ell+1)(\ell+2)}{r^{2}}+4 \omega\left(\frac{1}{\left(\omega r^{2}+2 \ell+3\right)}-\frac{2(2 \ell+3)}{\left(\omega r^{2}+2 \ell+3\right)^{2}}\right)-\omega\left(\ell+\frac{3}{2}\right),
$$

and the corresponding eigenfunctions can be easily obtained from those of $\psi_{n, m=1}^{(+)}$as given by Eq. (53).

As an illustration, we have calculated $I_{m=1}(r)$ in the case of $\omega=2$ and $\ell=1$ and is given by

$$
I_{1}(r)=-\frac{2 \exp \left(-r^{2}\right) r\left(15+4 r^{2}\left(5+r^{2}\right)\right)}{5 \sqrt{\pi}\left(3+2 r^{2}\right)}+\operatorname{erf}(r)
$$


where $\operatorname{erf}(r)$ is an error function. Using this expression of $I_{1}(r)$, one immediately obtains one continuous parameter family $\hat{V}^{(-)}(\lambda, r)$ of potentials which are strictly isospectral to the potential $V^{(-)}(r)$ as given by Eq. (55) in case $\omega=2$ and $l=1$ and provided if $\lambda>0$ or $\lambda<-1$. The corresponding eigenfunctions are then easily obtained from Eqs. (21) and (22).

One can also obtain the corresponding RE Pursey (26) and the RE AM (33) potentials by using the formula

$$
V_{1}^{[P]}(r)=V_{1}^{(-)}(r)-2 \frac{d^{2}}{d r^{2}} \ln \left(I_{1}(r)\right)
$$

and

$$
V_{1}^{[A M]}(r)=V_{1}^{(-)}(r)-2 \frac{d^{2}}{d r^{2}} \ln \left(I_{1}(r)-1\right)
$$

respectively, where $I_{1}(r)$ is as given by Eq. (58). The corresponding eigenfunctions can be easily obtained by setting $m=1$ in Eqs. (31) and (38) respectively.

Using the expression of $I_{1}(r)$ as given by Eq. (58), we have plotted some of the RE isospectral potentials and the corresponding ground state eigenfunctions in Figs 1(a) to 1(d). In particular, in Fig. 1(a), we have considered the variation of $\lambda$ from $+\infty$ to zero. In this case as $\lambda$ decreases from $+\infty$, the potential starts developing a minima and the corresponding position starts shifting towards $r=0$. The limiting case of $\lambda=0$ gives the corresponding rationally extended Pursey potential $V_{1}^{[P]}(r)$, whose solutions are associated with $X_{1}$ Laguerre EOPs. In Fig. $1(b)$, we have plotted the potentials for various values of $\lambda$ from $-\infty$ to -1 . As $\lambda$ increases from $-\infty$ and approaches -1 , the resulting attractive potential well shifts towards $r=\infty$ and in the limiting case of $\lambda=-1$, we get the rationally extended Abraham-Moses potential $V_{1}^{[A M]}(r)$. In Fig. $1(c)$ we have plotted the three strictly isospectral potentials namely the potential $V_{m=1}^{(+)}(r)$, the RE Pursey potential $V_{1}^{[P]}(r)$ and the RE Abraham-Moses potential $V_{1}^{[A M]}(r)$. The corresponding ground state eigenfunction for all the strictly isospectral RE potentials with positive $\lambda$ drawn in Fig. 1(a) are plotted in Fig. 1(d). 


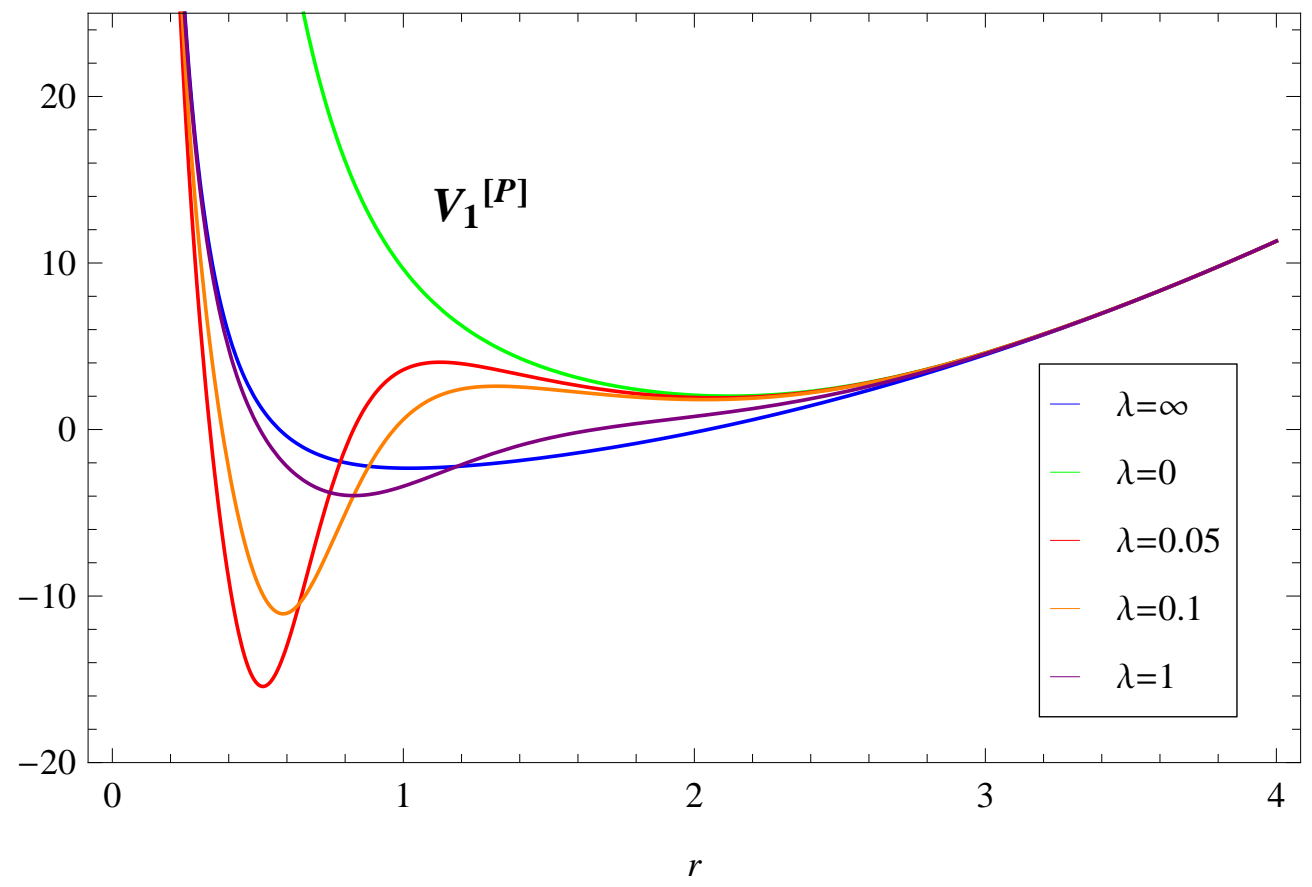

Fig.1: (a) Rationally extended potentials $\hat{V}_{1}(\lambda, r)$ strictly isospectral to the extended $3 D$ oscillator potential $V_{1}^{(-)}(r)$ for positive $\lambda=0,0.05,0.1,1$ and $\infty$. The extended Pursey potential is shown for $\lambda=0$.

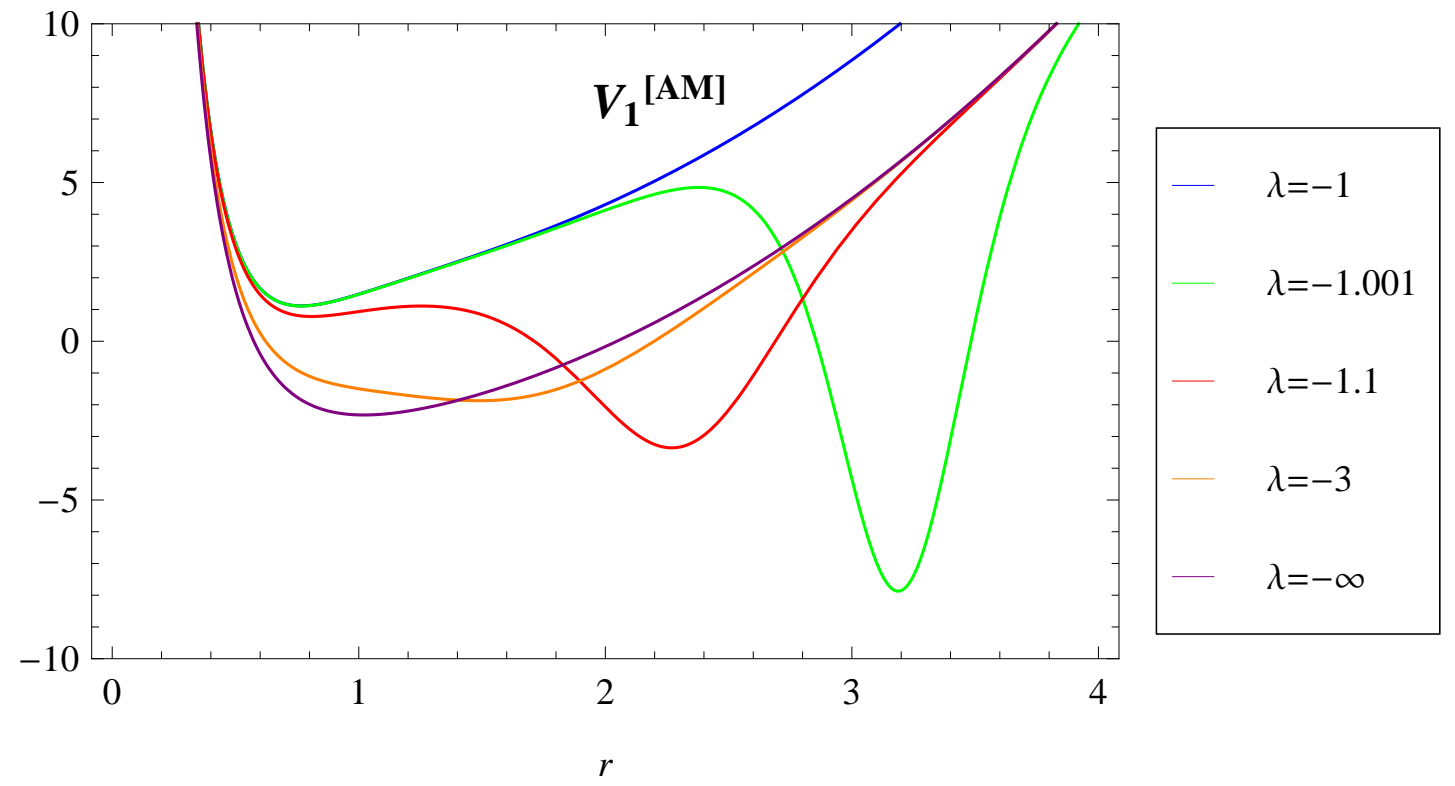

Fig.1: (b) Rationally extended potentials $\hat{V}_{1}(\lambda, r)$ for negative $\lambda=-\infty,-3,-1.1,-1.001$ and -1 . The extended AM potential is shown for $\lambda=-1$. 


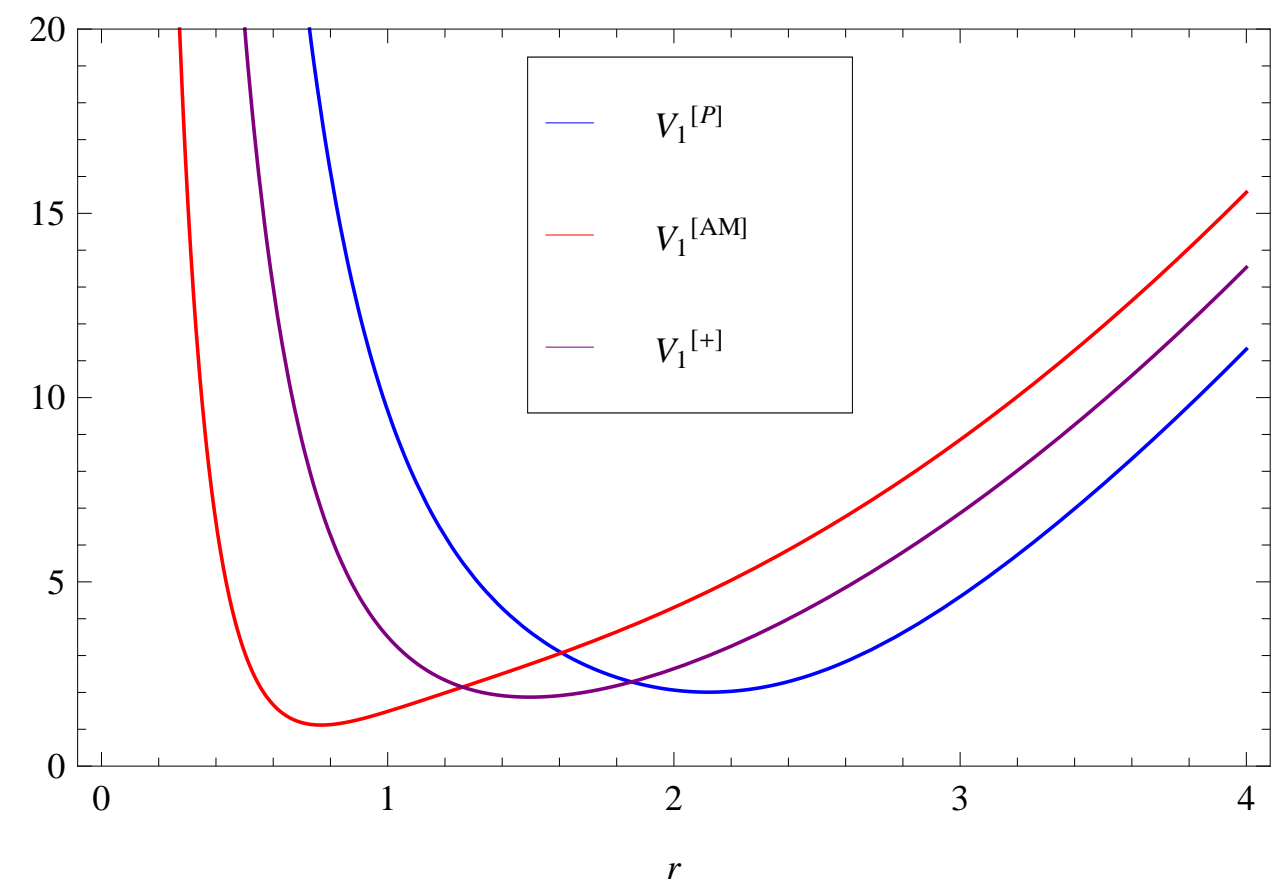

Fig.1: (c) The extended Pursey potential $V_{1}^{[P]}(r)$, extended $A M$ potential $V_{1}^{[A M]}(r)$ and the extended partner potentials $V_{1}^{(+)}(r)$.

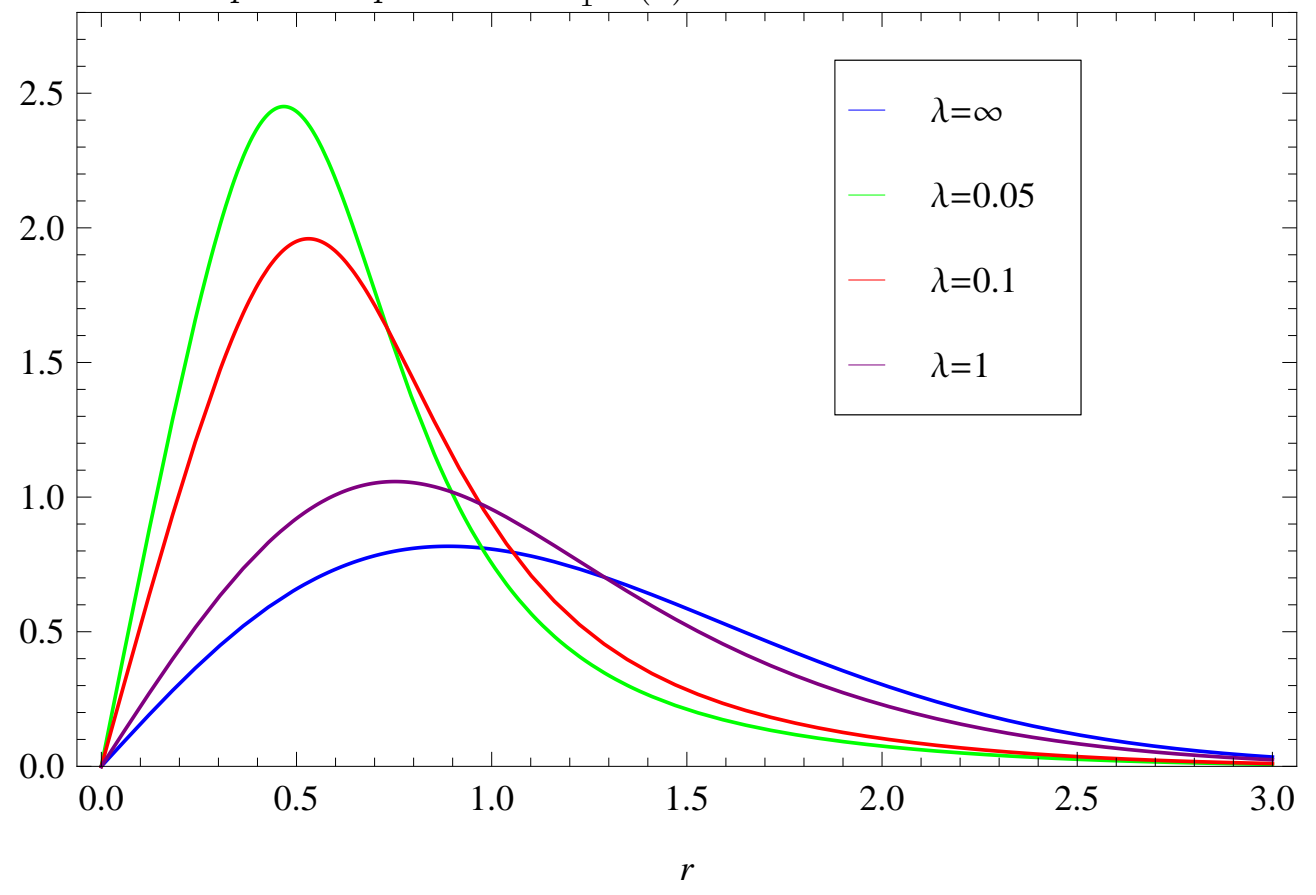

Fig.1: (d) Normalized ground-state wavefunctions $\frac{\hat{\psi}_{0,1}(\lambda, r)}{r}$ for some potentials (with positive $\lambda)$ shown in Fig. 1(a). 


\subsection{Rationally Extended Scarf-I potential}

In this case the terms in the superpotential (39) are defined as [28]

$$
W_{\text {con }}(x)=A \tan x-B \sec x
$$

and

$$
W_{m, r a t}(x)=-\frac{(\beta-\alpha+m-1)}{2} \cos x\left[\frac{P_{m-1}^{(-\alpha-1, \beta+1)}(z)}{P_{m}^{(-\alpha-2, \beta)}(z)}-\frac{P_{m-1}^{(-\alpha, \beta)}(z)}{P_{m}^{(-\alpha-1, \beta-1)}(z)}\right],
$$

where $\alpha=A-B-\frac{1}{2}, \quad \beta=A+B-\frac{1}{2}$ and $P_{m}^{(\alpha, \beta)}(z)$ (here $z=\sin x$ ) is a classical Jacobi polynomial. Using $W_{\text {con }}(x)$ and $W_{m, r a t}(x)$ in (40), we get the extended Scarf-I potential $V_{m}^{(-)}(x)$ with the terms

$$
V_{\text {con }}^{(-)}(x)=\left[(A-1) A+B^{2}\right] \sec ^{2} x-B(2 A-1) \sec x \tan x-A^{2}
$$

and

$$
\begin{aligned}
V_{m, \text { rat }}^{(-)}(x) & =(2 B-m-1)[2 A-1+(-2 B+1) \sin x]\left(\frac{P_{m-1}^{(-\alpha, \beta)}(\sin x)}{P_{m}^{(-\alpha-1, \beta-1)}(\sin x)}\right) \\
& +\frac{(-2 B-m+1)^{2}}{2} \cos ^{2} x\left(\frac{P_{m-1}^{(-\alpha, \beta)}(\sin x)}{P_{m}^{(-\alpha-1, \beta-1)}(\sin x)}\right)^{2} \\
& -2 m(-2 B-m-1) ; \quad-\pi / 2<x<\pi / 2, \quad 0<B<A-1,
\end{aligned}
$$

while the terms associated with the partner potential $V_{m}^{(+)}(x)$ are

$$
V_{\text {con }}^{(+)}(x)=\left[(A+1) A+B^{2}\right] \sec ^{2} x-B(2 A+1) \sec x \tan x-A^{2}
$$

and

$$
\begin{aligned}
V_{m, \text { rat }}^{(+)}(x) & =(2 B-m-1)[2 A+1+(-2 B+1) \sin x]\left(\frac{P_{m-1}^{(-\alpha-1, \beta+1)}(\sin x)}{P_{m}^{(-\alpha-2, \beta)}(\sin x)}\right) \\
& +\frac{(-2 B-m+1)^{2}}{2} \cos ^{2} x\left(\frac{P_{m-1}^{(-\alpha-1, \beta+1)}(\sin x)}{P_{m}^{(-\alpha-2, \beta)}(\sin x)}\right)^{2} \\
& -2 m(-2 B-m-1) .
\end{aligned}
$$

Using Eq. (4) the normalized ground state eigenfunction corresponding to the rationally extended Scarf-I potential $V_{m}^{(-)}(x)$ is given by

$$
\psi_{0, m}^{(-)}(x)=N_{m}^{(\alpha, \beta)} \frac{(1-\sin x)^{\frac{(A-B)}{2}}(1+\sin x)^{\frac{(A+B)}{2}}}{P_{m}^{(-\alpha-1, \beta-1)}(\sin x)} \hat{P}_{m}^{(\alpha, \beta)}(\sin x), \quad m=1,2 \ldots
$$

where the normalization constant

$$
N_{m}^{(\alpha, \beta)}=\left(\frac{(\alpha+1) \Gamma(\alpha+\beta+2)}{2^{\alpha+\beta+1}(\alpha-m+1)(m+\beta) \Gamma(\alpha+1) \Gamma(\beta)}\right)^{1 / 2} .
$$


The excited state eigenfunctions are also known [28] and are given by

$$
\psi_{n, m}^{(-)}(x)=N_{n, m}^{(\alpha, \beta)} \frac{(1-\sin x)^{\frac{(A-B)}{2}}(1+\sin x)^{\frac{(A+B)}{2}}}{P_{m}^{(-\alpha-1, \beta-1)}(\sin x)} \hat{P}_{n+m}^{(\alpha, \beta)}(\sin x)
$$

The energy eigenvalues are $(n=0,1,2, \ldots)$

$$
E_{n+1}^{(-)}=E_{n}^{+}=(A+n+1)^{2}-A^{2}, \quad E_{0}^{-}=0,
$$

while the normalization constant is

$$
N_{n, m}^{(\alpha, \beta)}=\left[\frac{n !(n+\alpha+1)^{2}(\alpha+\beta+2 n+1) \Gamma(n+\alpha+\beta+1)}{2^{\alpha+\beta+1}(n+\alpha-m+1)(n+m+\beta) \Gamma(n+\alpha+2) \Gamma(n+\beta)}\right]^{\frac{1}{2}} .
$$

The wavefunctions associated with the partner potentials can be obtained from Eq. (77) and gets

$$
\psi_{n, m}^{(+)}(x)=N_{n, m}^{(\alpha+1, \beta+1)} \frac{(1-\sin x)^{\frac{(A-B+1)}{2}}(1+\sin x)^{\frac{(A+B+1)}{2}}}{P_{m}^{(-\alpha-2, \beta)}(\sin x)} \hat{P}_{n+m}^{(\alpha+1, \beta+1)}(\sin x),
$$

where the exceptional Jacobi polynomial $\hat{P}_{n+m}^{(\alpha, \beta)}(z)$ in terms of the classical Jacobi polynomials is written as

$$
\begin{aligned}
\hat{P}_{n+m}^{(\alpha, \beta)}(z) & =(-1)^{m}\left[\left(\frac{1+\alpha+\beta+n}{1+\alpha+n}\right)\left(\frac{z-1}{2}\right) P_{m}^{(-\alpha-1, \beta-1)}(z) P_{n-1}^{(\alpha+2, \beta)}(z)\right. \\
& \left.+\left(\frac{1+\alpha-m}{1+\alpha+n}\right) P_{m}^{(-2-\alpha, \beta)}(z) P_{n}^{(\alpha+1, \beta-1)}(z)\right] .
\end{aligned}
$$

Using the normalized ground state eigenfunction as given by Eqs. (67), it is now straight forward to calculate the indefinite integral $I_{m}(x)$ by using the definition (18). Using this expression of $I_{m}(x)$ it is then straight forward to obtain one continuous parameter family of RE potentials $\hat{V}_{m}^{-}(\lambda, x)$ which are strictly isospectral to the potential $V_{m}^{-}(x)$. One can also obtain the corresponding RE Pursey (26) and the RE AM (33) potentials by using the Eqs. (59) and (60) . respectively. The corresponding eigenfunctions can be easily obtained by using Eqs. (31) and (38) respectively.

As an illustration, we now consider the $X_{1}$ case $(m=1)$ in detail. In this case the potentials and the wavefunctions are written as

$$
\begin{aligned}
V_{1}^{(-)}(x) & =\left[(A-1) A+B^{2}\right] \sec ^{2} x-B(2 A-1) \sec x \tan x \\
& +2\left(\frac{(2 A-1)}{(2 A-1-2 B \sin x)}-\frac{\left[(2 A-1)^{2}-B^{2}\right]}{(2 A-1-2 B \sin x)^{2}}\right)-A^{2}
\end{aligned}
$$

and

$$
\psi_{n, 1}^{(-)}(x)=N_{n, 1}^{(\alpha, \beta)} \frac{(1-\sin x)^{\frac{(A-B)}{2}}(1+\sin x)^{\frac{(A+B)}{2}}}{P_{1}^{(-\alpha-1, \beta-1)}(\sin x)} \hat{P}_{n+1}^{(\alpha, \beta)}(\sin x)
$$


respectively. The energy eigenvalues are same as that of the $X_{m}$ case (70). In this case, the partner potential (41) is given by

$$
\begin{aligned}
V_{1}^{(+)}(x) & =\left[(A+1) A+B^{2}\right] \sec ^{2} x-B(2 A+1) \sec x \tan x \\
& +2\left(\frac{(2 A+1)}{(2 A+1-2 B \sin x)}-\frac{\left[(2 A+1)^{2}-B^{2}\right]}{(2 A+1-2 B \sin x)^{2}}\right)-A^{2} .
\end{aligned}
$$

The associated wavefunction $\psi_{n, 1}^{(+)}(x)$ can also be easily obtain from Eq. (72). Using these results, the RE Pursey (26) and the extended AM (33) potentials with their corresponding wavefunctions (31) and (38) in terms of $X_{1}$ Jacobi EOPs can be find by setting $m=1$.

As an illustration, we have calculated $I_{m=1}(x)$ in the case of $A=3, B=1$ and is given by

$$
\begin{aligned}
I_{1}(x)=\frac{1}{2} & +\frac{1}{180 \pi(-5+2 \sin x)}[-900 x+675 \cos x+176 \cos 3 x+44 \cos 5 x \\
& +\cos 7 x+360 x \sin x-575 \sin 2 x-55 \sin 4 x+5 \sin 6 x]
\end{aligned}
$$

Using this expression of $I_{1}(x)$, one immediately obtains one continuous parameter family $\hat{V}_{1}^{(-)}(\lambda, x)$ of potentials which are strictly isospectral to the potential $V_{1}^{(-)}(x)$ as given by Eq. (174) in case $A=3, B=1$ and provided if $\lambda>0$ or $\lambda<-1$. The corresponding eigenfunctions are then easily obtained too. One can also obtain the corresponding RE Pursey (26) and the RE AM (33) potentials by using the Eqs. (159) and (60).

Using the above $I_{1}(x)$, we have plotted some of these extended isospectral potentials and normalized ground state wavefunctions in Figs. 2(a)-2(d). In this case as $\lambda$ varies from $\infty$ to 0 (Fig. 2(a)), the minima starts shifting towards $x=0$ and as $\lambda$ reduces to zero, we get the rationally extended Pursey potential. Similarly, in Fig. 2(b) we have plotted the potentials for negative $\lambda$ and as $\lambda$ approaches -1 , the minima shifts towards $x=\infty$. Ultimately as $\lambda=-1$, we get the rationally extended AM potential. Plots of three strictly isospectral potentials namely the RE partner potential $V_{1}^{(+)}(x)$, the RE Pursey potential $V_{1}^{[P]}$ and the RE AM potential $V_{1}^{[A M]}$ are shown in Fig. 2 (c). The normalized ground state eigenfunctions $\hat{\psi}_{0,1}^{(-)}(x)$ of all the potentials with positive $\lambda$ as shown in Fig. 2 (a) are also plotted in Fig. 2 (d). 


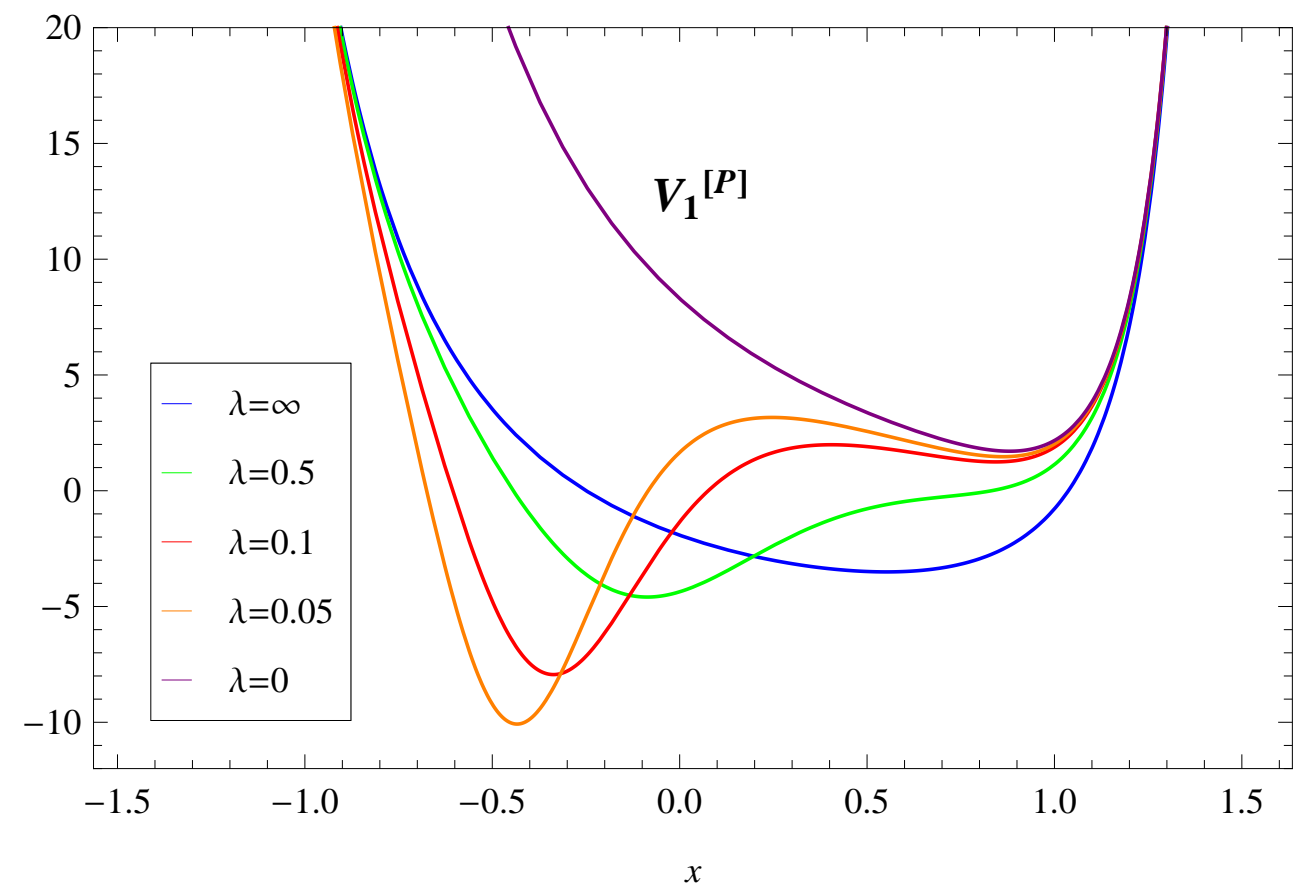

Fig.2: (a) Rationally extended potentials $\hat{V}_{1}(\lambda, x)$ strictly isospectral to the extended ScarfI potential $V_{1}^{(-)}(x)$ for positive $\lambda=0,0.05,0.1,0.5$ and $\infty$.

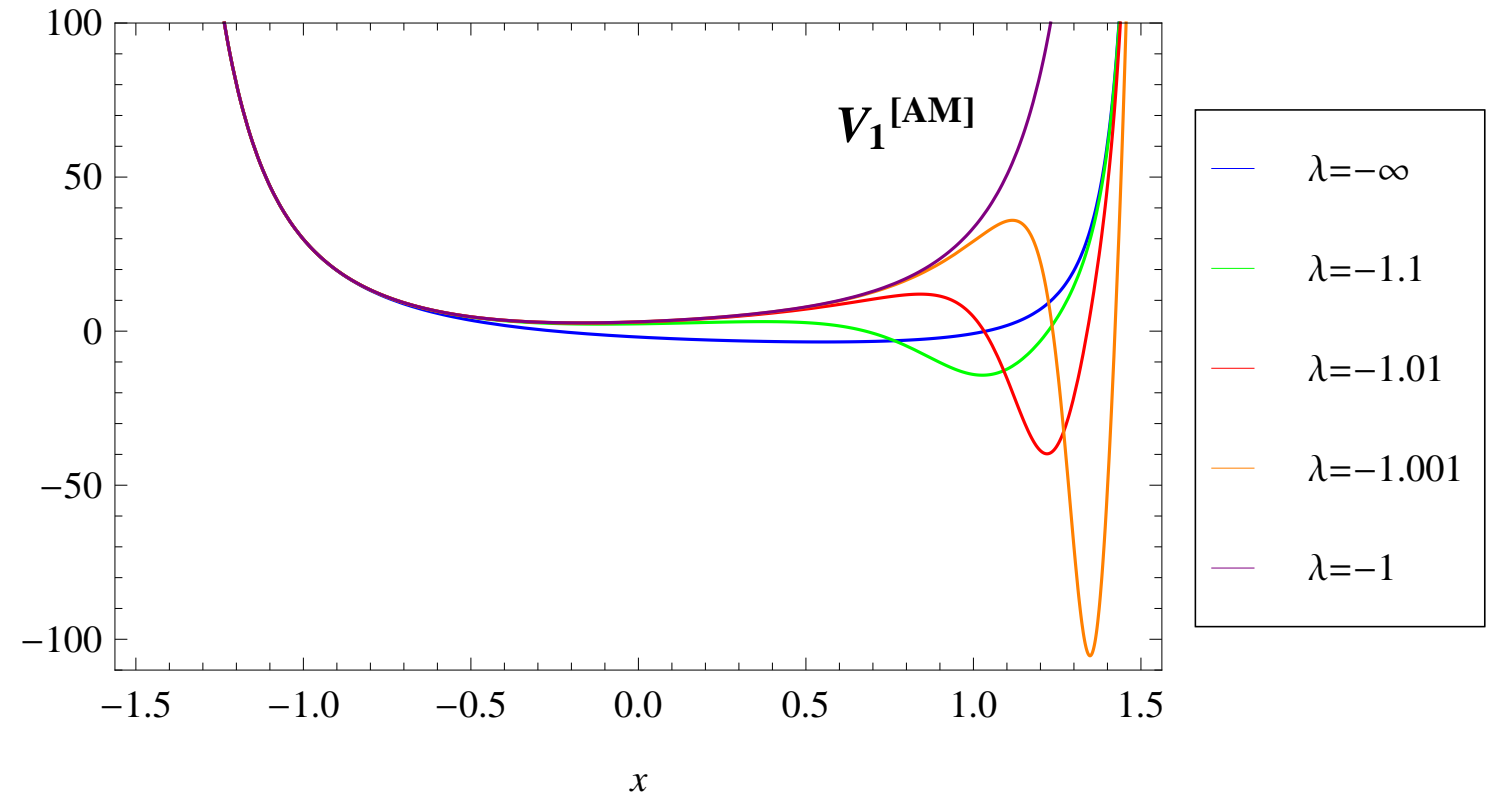

Fig.2: (b) Rationally extended potentials $\hat{V}_{1}(\lambda, x)$ for negative $\lambda=-\infty,-1.1,-1.01,-1.001$ and -1 . 


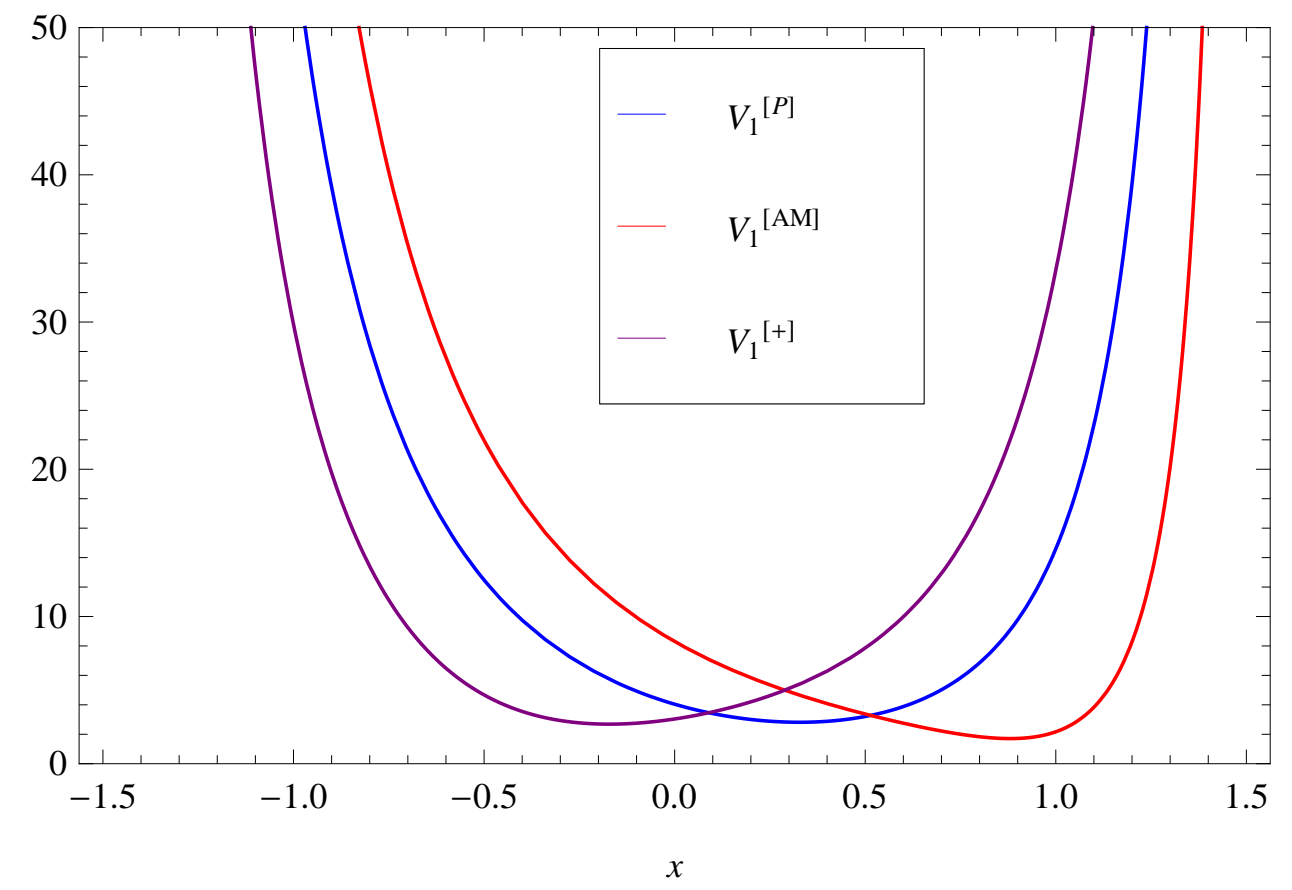

Fig.2: (c) The extended Pursey potential $V_{1}^{[P]}(x)$, extended AM potential $V_{1}^{[A M]}(x)$ and the extended partner potentials $V_{1}^{(+)}(x)$.

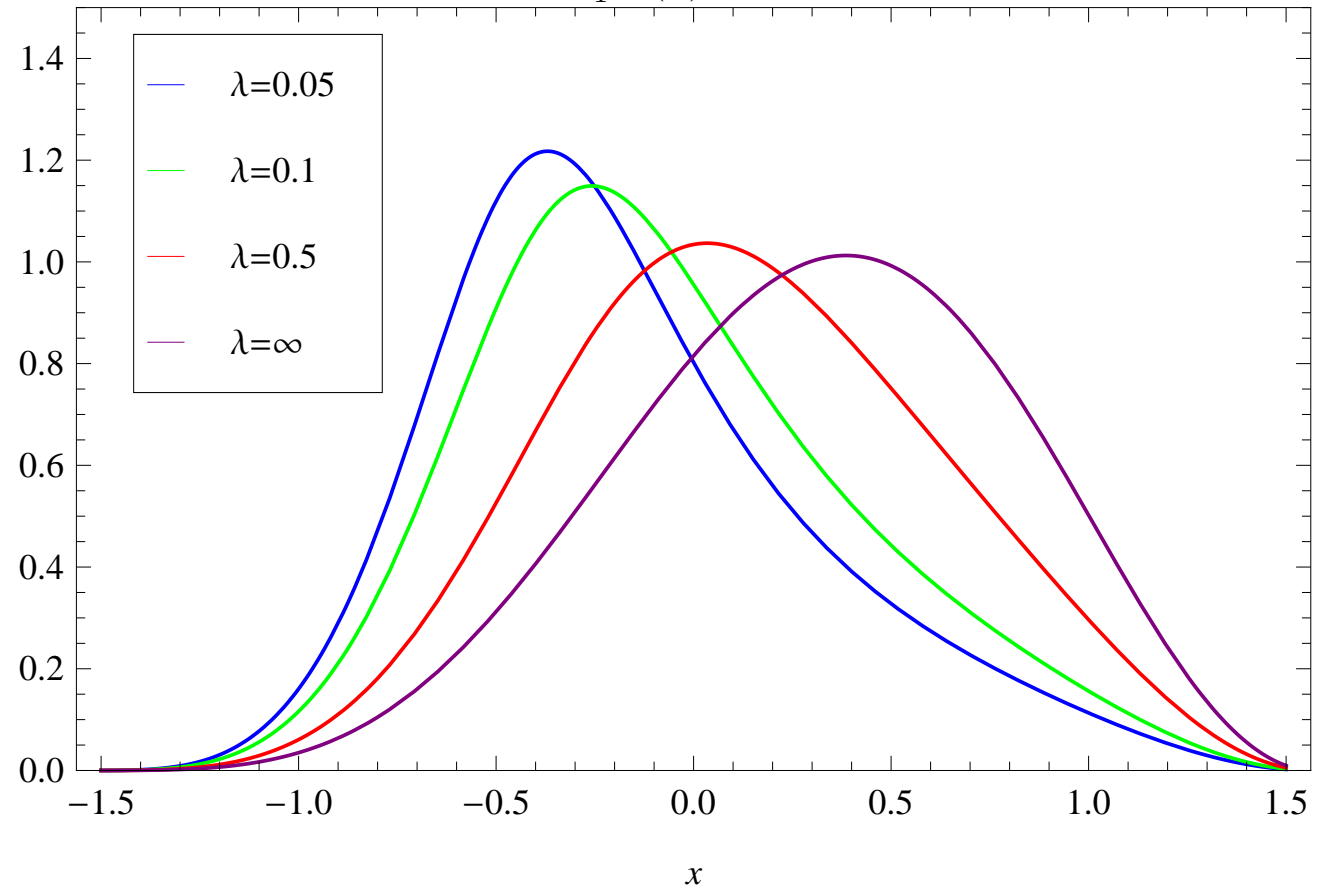

Fig.2:(d) Normalized ground-state eigenfunctions $\hat{\psi}_{0,1}(\lambda, x)$ for some of the potentials with positive $\lambda$ as shown in Fig. 2(a). 


\subsection{Rationally Extended generalized Poschl-Teller (GPT) po- tential}

As a last example, we consider the RE GPT potential which has both the bound as well as the scattering state solutions. The terms corresponding to the superpotential (39) associated with this potential are given as

$$
W_{\text {con }}(x)=A \operatorname{coth} x-B \operatorname{cosech} x
$$

and

$$
W_{m, r a t}(x)=-\frac{(\beta-\alpha+m-1)}{2} \sinh x\left[\frac{P_{m-1}^{(-\alpha-1, \beta+1)}(z)}{P_{m}^{(-\alpha-2, \beta)}(z)}-\frac{P_{m-1}^{(-\alpha, \beta)}(z)}{P_{m}^{(-\alpha-1, \beta-1)}(z)}\right],
$$

where $\alpha=B-A-\frac{1}{2}, \quad \beta=-B-A-\frac{1}{2}$ and $z=\cosh x$. Using (78) and (79) in (40), we get the extended GPT potential $V_{m}^{(-)}(x)$ with the terms

$$
V_{\text {con }}^{(-)}(x)=\left[(A+1) A+B^{2}\right] \operatorname{cosech}^{2} x-B(2 A+1) \operatorname{cosech} x \operatorname{coth} x+A^{2}
$$

and

$$
\begin{aligned}
V_{m, \text { rat }}^{(-)}(x) & =-(2 B-m+1)[2 A+1-(2 B+1) \cosh x]\left(\frac{P_{m-1}^{(-\alpha, \beta)}(\cosh x)}{P_{m}^{(-\alpha-1, \beta-1)}(\cosh x)}\right) \\
& +\frac{(2 B-m+1)^{2}}{2} \sinh ^{2} x\left(\frac{P_{m-1}^{(-\alpha, \beta)}(\cosh x)}{P_{m}^{(-\alpha-1, \beta-1)}(\cosh x)}\right)^{2} \\
& +2 m(-2 B-m-1) ; \quad 0 \leq x \leq \infty, \quad B>A+1>1,
\end{aligned}
$$

while the terms associated with the partner potential $V_{m}^{(+)}(x)$ are

$$
V_{\text {con }}^{(+)}(x)=\left[A(A-1)+B^{2}\right] \operatorname{cosech}^{2} x-B(2 A-1) \operatorname{cosech} x \operatorname{coth} x+A^{2}
$$

and

$$
\begin{aligned}
V_{m, \text { rat }}^{(+)}(x) & =-(2 B-m+1)[2 A+1-(2 B+1) \cosh x]\left(\frac{P_{m-1}^{(-\alpha-1, \beta+1)}(\cosh x)}{P_{m}^{(-\alpha-2, \beta)}(\cosh x)}\right) \\
& +\frac{(2 B-m+1)^{2}}{2} \sinh ^{2} x\left(\frac{P_{m-1}^{(-\alpha-1, \beta+1)}(\cosh x)}{P_{m}^{(-\alpha-2, \beta)}(\cosh x)}\right)^{2} \\
& +2 m(-2 B-m-1) .
\end{aligned}
$$

The normalized ground state eigenfunction corresponding to the rationally extended GPT potential $V_{m}^{(-)}(x)$ is easily calculated and is given by

$$
\psi_{0, m}^{(-)}(x)=N_{m}^{(\alpha, \beta)} \frac{(\cosh x-1)^{\frac{(B-A)}{2}}(\cosh x+1)^{-\frac{(B+A)}{2}}}{P_{m}^{(-\alpha-1, \beta-1)}(\cosh x)} \hat{P}_{m}^{(\alpha, \beta)}(\cosh x), \quad m=1,2 \ldots
$$


where the normalization constant

$$
N_{m}^{(\alpha, \beta)}=\left[\frac{(-\alpha-\beta-1)(\alpha+1) \Gamma(-\beta+1)}{2^{\alpha+\beta+1}(-\beta-m)(\alpha-m+1) \Gamma(\alpha+1) \Gamma(-\alpha-\beta)}\right]^{1 / 2} .
$$

The excited state solutions are also known [35] and given as

$$
\psi_{n, m}^{(-)}(x)=N_{n, m}^{(\alpha, \beta)} \frac{(\cosh x-1)^{\frac{(B-A)}{2}}(\cosh x+1)^{-\frac{(B+A)}{2}}}{P_{m}^{(-\alpha-1, \beta-1)}(\cosh x)} \hat{P}_{n+m}^{(\alpha, \beta)}(\cosh x), \quad m=1,2 \ldots
$$

The energy eigenvalues are given by $(n=0,1,2)$

$$
E_{n+1}^{(-)}=E_{n}^{(+)}=A^{2}-(A-n-1)^{2}, \quad E_{0}^{(-)}=0,
$$

where the normalization constant

$$
N_{n, m}^{(\alpha, \beta)}=\left[\frac{n !(-\alpha-\beta-2 n-1)(n+\alpha-m+1)(\alpha+n+1) \Gamma(-\beta-n+1)}{2^{\alpha+\beta+1}(-\beta-n-m)(\alpha-m+1)^{2} \Gamma(\alpha+n+1) \Gamma(-\alpha-\beta-n)}\right]^{1 / 2} .
$$

The wavefunctions corresponding to the partner potential can be obtain using Eq. (7) i.e, $\psi_{n, m}^{(+)}(x)=N_{n, m}^{(\alpha+1, \beta+1)} \frac{(\cosh x-1)^{\frac{(B-A+1)}{2}}(\cosh x+1)^{-\frac{(B+A-1)}{2}}}{P_{m}^{(-\alpha-2, \beta)}(\cosh x)} \hat{P}_{n+m}^{(\alpha+1, \beta+1)}(\cosh x), \quad m=1,2 \ldots$

The scattering amplitudes ( $s$-wave) related to the potential $V_{m}^{(-)}(x)$ are well known [35] and are given by

$$
\begin{aligned}
s_{m}^{(-)}\left(k^{\prime}\right) & =2^{-4 i k^{\prime}} \frac{\Gamma\left(2 i k^{\prime}\right) \Gamma\left(-A-i k^{\prime}\right) \Gamma\left(B-i k^{\prime}+1 / 2\right)}{\Gamma\left(-2 i k^{\prime}\right) \Gamma\left(-A+i k^{\prime}\right) \Gamma\left(B+i k^{\prime}+1 / 2\right)} \\
& \times\left[\frac{\left[B^{2}-\left(i k^{\prime}-1 / 2\right)^{2}\right]+\left(B-i k^{\prime}+1 / 2\right)(1-m)}{\left[B^{2}-\left(i k^{\prime}+1 / 2\right)^{2}\right]+\left(B+i k^{\prime}+1 / 2\right)(1-m)}\right], \quad m \geq 1 .
\end{aligned}
$$

Just as in the last two examples, it is now straight forward to calculate $I_{m}(x)$ and hence one continuous parameter family of potentials $\hat{V}_{m}^{(-)}(\lambda, x)$ which are strictly isospectral to the RE GPT potential $V_{m}^{(-)}(x)$ in case $\lambda>0$ or $\lambda<-1$. Further, The RE Pursey and the RE AM potentials for this case can also be obtained in a straightforward way in case $\lambda=0$ or $\lambda=-1$ respectively. .

From Eqs. (78) and (179) as $x \rightarrow+\infty$, we get the asymptotic form of the superpotentials

$$
W_{\text {con }}(x \rightarrow+\infty)=A, \quad W_{m, r a t}(x \rightarrow+\infty)=0,
$$

hence the superpotential $W_{m}(x)$ becomes

$$
W_{m}(x \rightarrow+\infty)=A .
$$


Using Eqs. (11), (30) and (37), the scattering amplitudes of the partner potential, the extended Pursey and the extended AM potentials are related to the scattering amplitude of the $V_{m}^{(-)}(x)$ by

$$
\begin{aligned}
s_{m}^{(+)}\left(k^{\prime}\right) & =\left(\frac{A+i k^{\prime}}{A-i k^{\prime}}\right) s_{m}^{(-)}\left(k^{\prime}\right) \\
s_{m}^{[P]}\left(k^{\prime}\right) & =s_{m}^{(-)}\left(k^{\prime}\right)
\end{aligned}
$$

and

$$
s_{m}^{[A M]}\left(k^{\prime}\right)=\left(\frac{A+i k^{\prime}}{A-i k^{\prime}}\right)^{2} s_{m}^{(-)}\left(k^{\prime}\right)
$$

respectively. Thus unlike the two examples discussed above where the spectrum was purely discrete and hence $V_{m}^{(+)}, V_{m}^{[P]}$ and $V_{m}^{[A M]}$ were strictly isospectral, for RE GPT case, the three are isospectral but not strictly isospectral.

We now illustrate the above results by discussing in detail the $X_{1}$ case for which the potentials and the eigenfunctions are

$$
\begin{aligned}
V_{1}^{(-)}(x) & =\left(B^{2}+A(A+1)\right) \operatorname{cosech}^{2} x-B(2 A+1) \operatorname{cosech} x \operatorname{coth} x \\
& +2\left[\frac{(2 A+1)}{(2 B \cosh x-2 A-1)}-\frac{\left(4 B^{2}-(2 A+1)^{2}\right)}{(2 B \cosh x-2 A-1)^{2}}\right]+A^{2} .
\end{aligned}
$$

and

$$
\psi_{n, 1}^{(-)}(x)=N_{n, 1}^{(\alpha, \beta)} \frac{(\cosh x-1)^{\frac{(B-A)}{2}}(\cosh x+1)^{-\frac{(B+A)}{2}}}{P_{1}^{(-\alpha-1, \beta-1)}(z)} \hat{P}_{n+1}^{(\alpha, \beta)}(z)
$$

respectively. As usual the energy eigenvalues are same as that of the $X_{m}$ case (87). The partner potential corresponding to this case are

$$
\begin{aligned}
V_{1}^{(+)}(x) & =\left(B^{2}+A(A-1)\right) \operatorname{cosech}^{2} x-B(2 A-1) \operatorname{cosech} x \operatorname{coth} x \\
& +2\left[\frac{(2 A-1)}{(2 B \cosh x-2 A+1)}-\frac{\left(4 B^{2}-(2 A-1)^{2}\right)}{(2 B \cosh x-2 A+1)^{2}}\right]+A^{2}
\end{aligned}
$$

and the eigenfunction can be obtained from Eq. (89). The scattering amplitude (90) for the $X_{1}$ case is given by

$$
s_{1}^{(-)}\left(k^{\prime}\right)=2^{-4 i k^{\prime}} \frac{\Gamma\left(2 i k^{\prime}\right) \Gamma\left(-A-i k^{\prime}\right) \Gamma\left(B-i k^{\prime}+1 / 2\right)\left(B^{2}-\left(i k^{\prime}-1 / 2\right)^{2}\right)}{\Gamma\left(-2 i k^{\prime}\right) \Gamma\left(-A+i k^{\prime}\right) \Gamma\left(B+i k^{\prime}+1 / 2\right)\left(B^{2}-\left(i k^{\prime}+1 / 2\right)^{2}\right)} .
$$

Following the same procedure as discussed in the last two examples it is straight forward tp calculate the integral $I_{1}(x)$. As an illustration, we have calculated it in case $A=1, B=3$ and we find

$$
I_{1}(x)=\left[\frac{(3+11 \cosh x+\cosh 2 x) \operatorname{sech}^{2} \frac{x}{2} \tanh ^{5} \frac{x}{2}}{(-2+4 \cosh x)}\right]
$$

so that now it is straight forward to obtain the corresponding one parameter family of strictly isospectral potentials $\hat{V}_{1}^{(-)}(\lambda, x)$ in case $\lambda>0$ or $\lambda<-1$ and also obtain the 
corresponding eigenfunctions. Further, for $\lambda=0,-1$ it is straight forward to obtain the corresponding extended Pursey and Abraham-Moses potentials. In Figs. 3 (a) and 3(b), we have shown the plots of different $\mathrm{RE}$ isospectral potentials in case $\lambda \geq 0$ and $\lambda \leq-1$ respectively. In Fig. 3 (c) we have shown the plots of the three isospectral RE potentials $V_{1}^{(+)}, V_{1}^{[P]}$ and $V_{1}^{[A M]}$ while in Fig. $3(\mathrm{~d})$ we have shown the plots of the ground state eigenfunctions of $\hat{V}^{(-)}(\lambda, x)$ in the case of few positive values of $\lambda$.

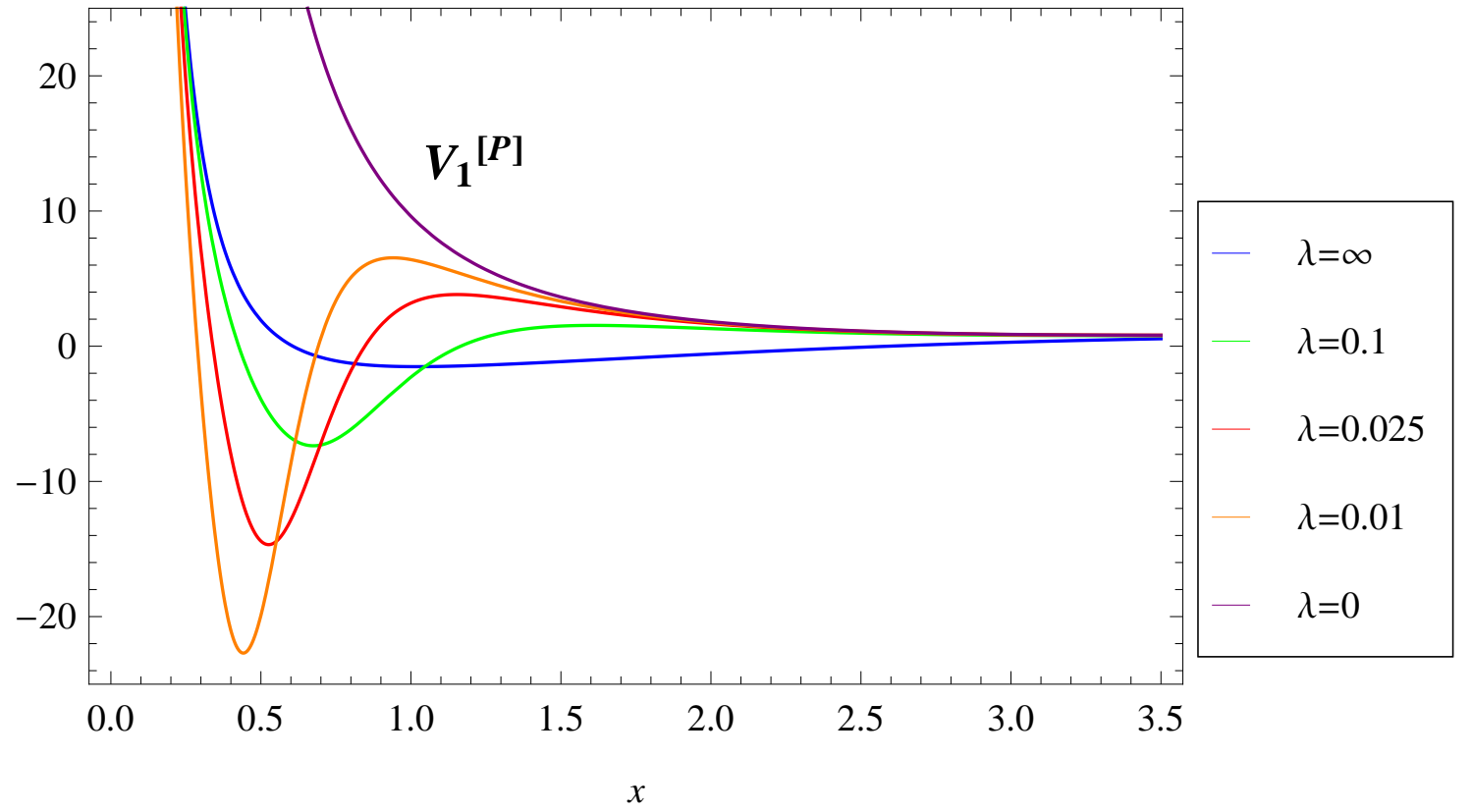

Fig.3: (a) Rationally extended potentials $\hat{V}_{1}(\lambda, x)$ strictly isospectral to the extended GPT potential $V_{1}^{(-)}(x)$ for $\lambda=0,0.01,0.025,0.1$ and $\infty$.

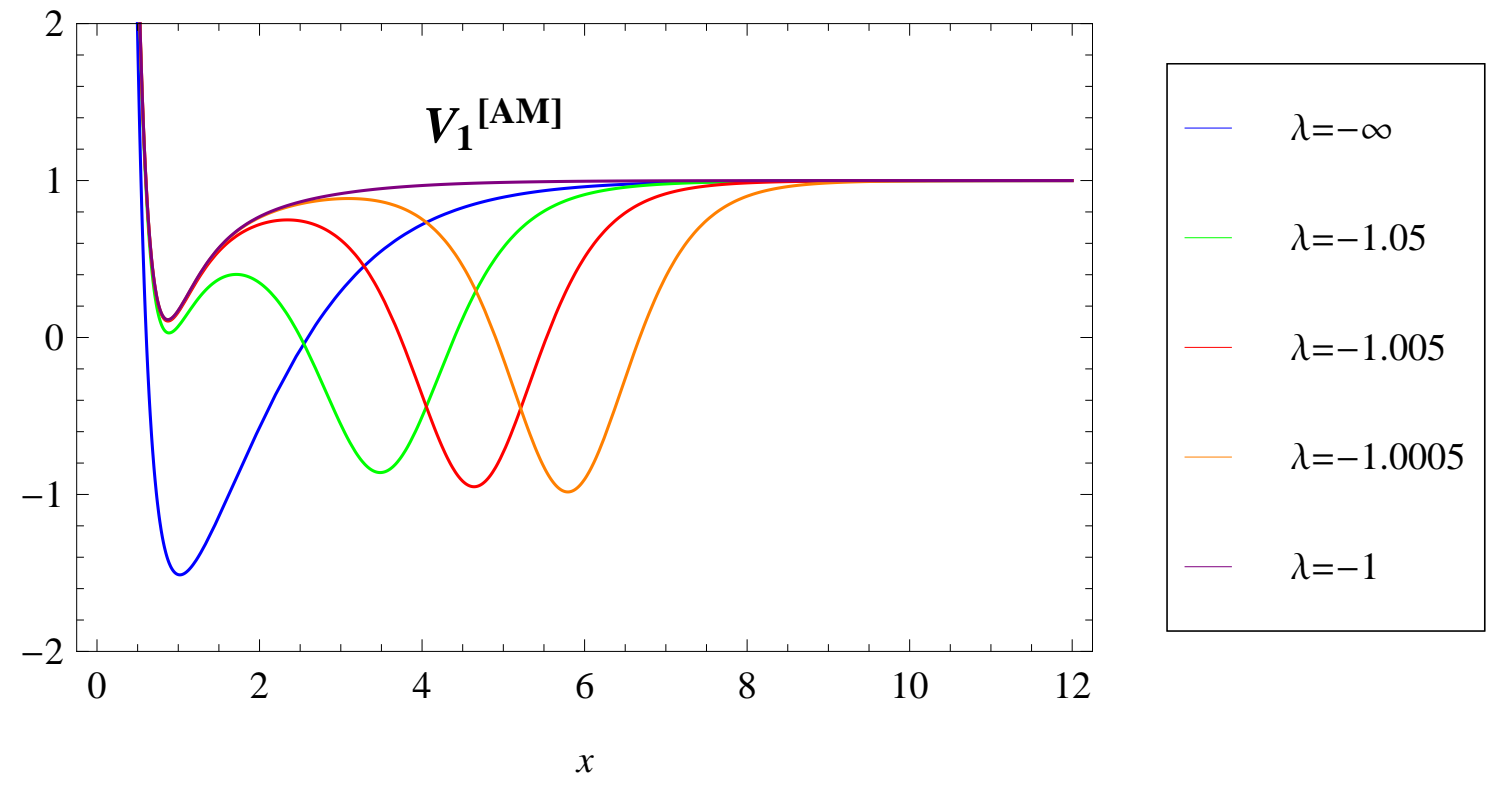

Fig.3: (b) Rationally extended potentials $\hat{V}_{1}(\lambda, x)$ for negative $\lambda=-\infty,-1.05,-1.005,-1.0005$ 


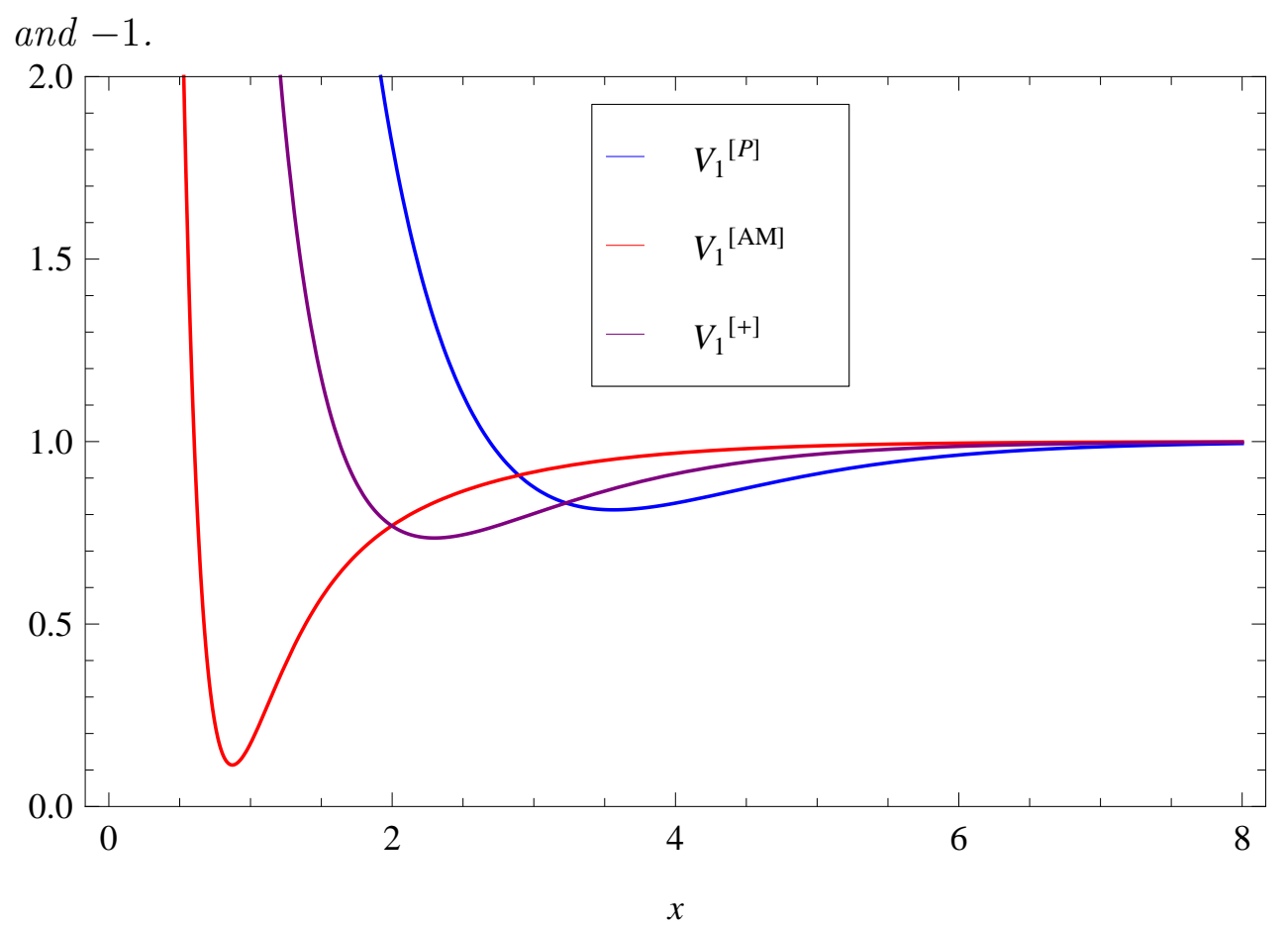

Fig.3: (c) The extended Pursey potential $V_{1}^{[P]}(x)$, extended AM potential $V_{1}^{[A M]}(x)$ and the extended partner potentials $V_{1}^{(+)}(x)$.

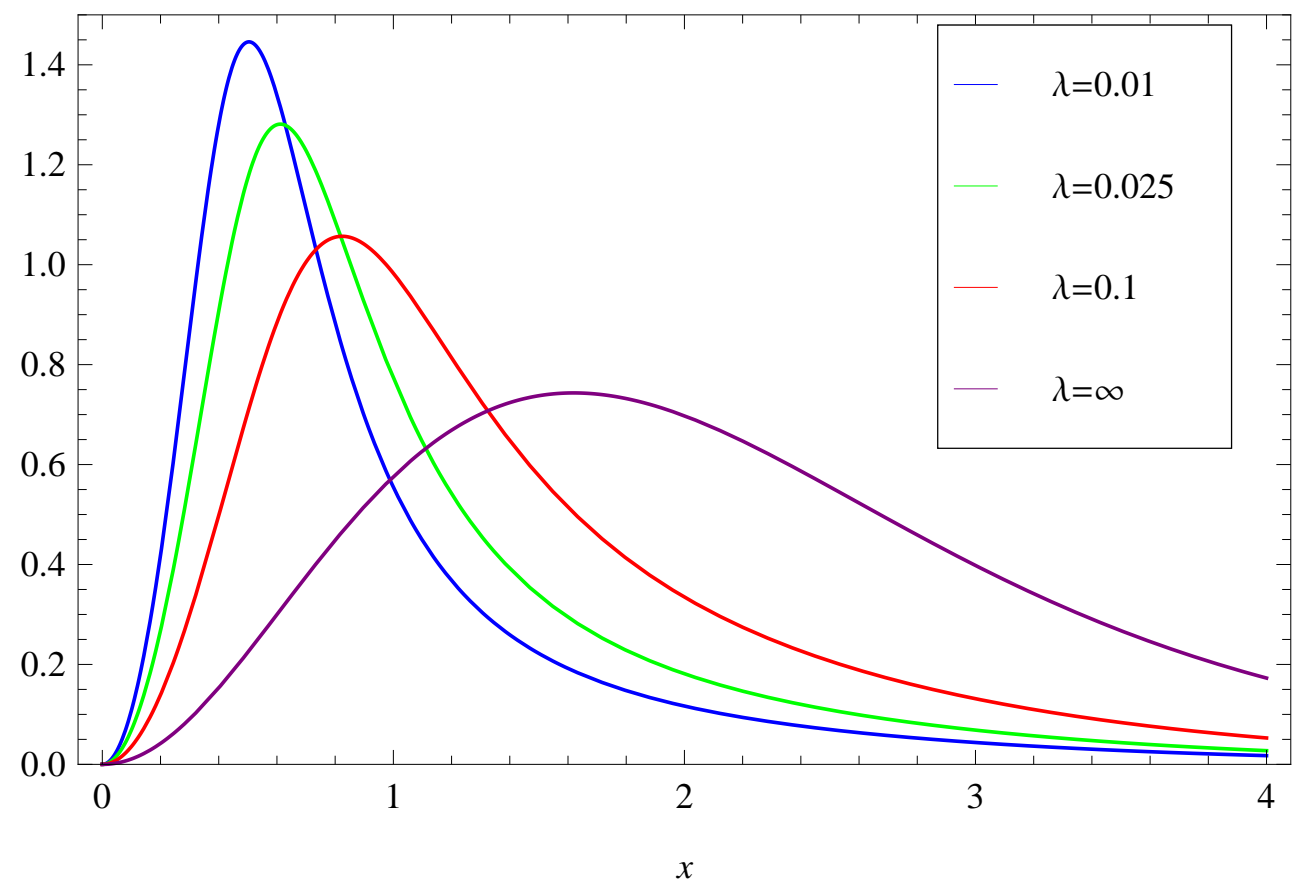

Fig.3: (d) Normalized ground-state eigenfunctions $\hat{\psi}_{0,1}(\lambda, x)$ for all the potentials with positive $\lambda$ shown in Fig. 3(a). 
The scattering amplitudes $s_{1}^{(-)}\left(k^{\prime}\right)$ (97) becomes

$$
s_{1}^{(-)}\left(k^{\prime}\right)=2^{-4 i k^{\prime}} \frac{\Gamma\left(2 i k^{\prime}\right) \Gamma\left(-1-i k^{\prime}\right) \Gamma\left(3-i k^{\prime}+1 / 2\right)\left(9-\left(i k^{\prime}-1 / 2\right)^{2}\right)}{\Gamma\left(-2 i k^{\prime}\right) \Gamma\left(-1+i k^{\prime}\right) \Gamma\left(3+i k^{\prime}+1 / 2\right)\left(9-\left(i k^{\prime}+1 / 2\right)^{2}\right)} .
$$

Thus the scattering amplitudes (93) corresponding to the partner potential, Pursey and AM potentials are given as

$$
\begin{aligned}
s_{1}^{(+)}\left(k^{\prime}\right) & =\left(\frac{1+i k^{\prime}}{1-i k^{\prime}}\right) s_{1}^{(-)}\left(k^{\prime}\right) \\
s_{1}^{[P]}\left(k^{\prime}\right) & =s_{1}^{(-)}\left(k^{\prime}\right)
\end{aligned}
$$

and

$$
s_{1}^{[A M]}\left(k^{\prime}\right)=\left(\frac{1+i k^{\prime}}{1-i k^{\prime}}\right)^{2} s_{1}^{(-)}\left(k^{\prime}\right)
$$

respectively.

\section{Conclusions}

In this paper we have considerably extended the list of the exactly solvable rationally extended potentials. In particular, by starting from three conventional (non-rational) potentials, we have obtained two parameter family of exactly solvable strictly isospectral RE potentials by using the formalism of SUSY Quantum mechanics. Out of these two parameters, one, i.e. $\lambda$ is a continuous parameter while $m$ is a discrete parameter $(m=1,2,3, \ldots)$ corresponding to $X_{m}$ EOPs. We considered three RE potentials namely the RE radial oscillator, RE Scarf-I and the RE generalized Pöschl-Teller potentials as starting potentials and generated one continuous parameter family of strictly isospectral RE potentials corresponding to these potentials. Further, we have also obtained the corresponding bound state eigenfunctions in terms of the $X_{m}$ EOPs. Besides, in the case of the RE GPT, we have also obtained the scattering amplitude of these potentials. In addition, in all these cases we have also obtained the corresponding RE partner potential $V_{m}^{(+)}$, the RE Pursey potential $V_{m}^{[P]}$ and the RE Abraham-Moses potential $V_{m}^{[A M]}$ and their bound state eigenfunctions in terms of $X_{m}$ EOPs. It is worth pointing out that while these three potentials are strictly isospectral in the case of the RE radial oscillator and the RE Scarf-I, they are only isospectral (but not strictly isospectral) in the case of the RE generalized Pöschl-Teller potential. As an illustration, we have considered the $X_{1}$ case in detail and shown the behavior of these extended potentials as well as the behaviour of the corresponding normalized ground state wavefunctions for different values (positive as well as negative) of $\lambda$.

Finally, by starting from the ground state of each of these new potentials and repeating exactly the same procedure as adopted in this paper, in each case we can further enlarge the number of exactly solvable RE potentials by obtaining in each case yet another one 
continuous parameter family of strictly isospectral potentials as well as new RE Pursey and RE Abraham-Moses potentials.

\section{Acknowledgments}

BPM acknowledges the support from MATRIX project (Grant No. MTR/2018/000611), SERB, DST Govt. of India. AK is grateful to Indian National Science Academy (INSA) for awarding INSA senior scientist position at Savitribai Phule Pune University.

\section{References}

[1] F. Cooper, A. Khare, U. Sukhatme Phys. Rep. 251 (1995) 267; "SUSY in Quantum Mechanics" World Scientific (2001).

[2] A. Khare and U. P. Sukhatme J. Phys. A: Math. Gen.22(1989) 2847

[3] Z. S. Agronorich and V. A. Marchenko, "The Inverse Problem of Scattering Theory" Gordon and Breach, New York (1963).

[4] K. Chandan and P. C. Sabatier, "Inverse Problem in Quantum Scattering Theory" Springer, New York (1977).

[5] D Baye, Phys. Rev. Lett. 58 (1987) 2738.

[6] G. L. Lamb, D Baye, "Elements of Soliton Theory" Springer, New York (1980).

[7] P. G. Drazin and R. S. Johson, "Solitons: An Introduction" Cambridge University Press (1989).

[8] M.M. Nieto, Phys. Lett. B 145 (1984) 208.

[9] R. D. Amado, Phys. Rev. A 37 (1988) 2277.

[10] D. L. Pursey, Phys. Rev. D 331048 (1986) 2267.

[11] C. V. Sukumar, J. Phys. A: Math. Gen. 21 (1988) L455; J. Phys. A: Math. Gen. 18 (1985) 2937.

[12] P. B. Abraham and H.E. Moses, Phys. Rev. A 22 (1980) 1333.

[13] G. Darboux, C. R. Acad. Sci. (Paris) 94 (1882) 1456.

[14] A. Khare and U. Sukhatme, J. Phys. A: Math. Gen. 22 (1989) 2847.

[15] I. M. Gelfand and B. M. Levitan, Am. Math. Soc. Transl. 1 (1951) 253.

[16] D. Gomez-Ullate, N. Kamran and R. Milson, J. Math. Anal.Appl. 359 (2009) 352.

[17] D. Gomez-Ullate, N. Kamran and R. Milson, J. Phys. A 43 (2010) 434016. 
[18] D. Gomez-Ullate, N. Kamran and R. Milson, Contemp. Math. 563 (2012) 51.

[19] C. Quesne, J.Phys.A 41 (2008) 392001.

[20] B. Bagchi, C. Quesne and R. Roychoudhary, Pramana J. Phys. 73(2009) 337, C. Quesne, SIGMA 5 (2009) 84.

[21] S. Odake and R. Sasaki, Phys. Lett. B, 684 (2010) 173; ibid 679 (2009) 414. J. Math. Phys, 51, 053513 (2010).

[22] C-L. Ho, S ODAKE and R Sasaki, SIGMA 7 (2011) 107.

[23] C-L. Ho and R Sasaki, ISRN Math. Phys. (2012) 920475.

[24] C. Quesne, Int. J. Mod. Phys. A 26 (2011) 5337.

[25] Y. Grandati, J. Math. Phys. 52103505 (2011).

[26] Y. Grandati, Ann. Phys. 3262074 (2011); 3272411 (2012); 327185 (2012).

[27] C. Quesne, SIGMA 8080 (2012).

[28] B Midya and B Roy, J. Phys. A: Mathematical and Theoretical 46 (17) 175201 (2013).

[29] R. K. Yadav, B. P. Mandal and A. Khare, Acta Polytechnica 57(6) (2017) 477.

[30] B Midya and B Roy, Phys. Lett. A 373 (45) (2009) 4117.

[31] B Midya, B Roy and T. Tanaka, J. Phys. A 45 (2012) 205303.

[32] C.-L Ho, Ann. Phys. 326 (2011) 797.

[33] D. Dutta and P. Roy, J. Math. Phys.52 (2011) 032104.

[34] R. K. Yadav, A. Khare and B. P. Mandal, Ann. Phys. 331 (2013) 313.

[35] R. K. Yadav, A. Khare and B. P. Mandal, Phys. Lett. B 723433 (2013); Phys. Lett. A 379 (2015) 67.

[36] C. L. Ho, J. C. Lee and R. Sasaki, Annals of Physics 343 (2014) 115.

[37] R. K. Yadav, N. Kumari, A. Khare and B. P. Mandal, Ann. Phys. 359 (2015) 46.

[38] N. Kumari, R. K. Yadav, A. Khare, B. Bagchi and B. P. Mandal, Ann. Phys.373 (2016) 163.

[39] A. Ramos et al., Ann. Phys.382 (2017) 143.

[40] R. K. Yadav, A. Khare, N. Kumari, B. Bagchi and B. P. Mandal, J. Math. Phys.57 (2016) 062106. 
[41] N. Kumari, R. K. Yadav, A. Khare and B. P. Mandal, Ann. Phys.385 (2017) 57.

[42] N. Kumari, R. K. Yadav, A. Khare and B. P. Mandal, J. Math. Phys.59 (2018) 062103-1.

[43] B. Basu-Mallick, B. P. Mandal and P. Roy, Ann. Phys.380 (2017) 206.

[44] R. K. Yadav, A. Khare, N. Kumari and B. P. Mandal, Ann. Phys.400 (2019) 189. 